KA-TP-30-1996

$\mathrm{PM} / 96-39$

December 1996

\title{
The Higgs-photon-Z boson Coupling Revisited
}

\author{
A. Djouadi ${ }^{1,2}$, V. Driesen ${ }^{2}$, W. Hollik ${ }^{2}$ and A. Kraft ${ }^{2}$ \\ ${ }^{1}$ Physique Mathématique et Théorique, UPRES-A 5032, \\ Université de Montpellier II, F-34095 Montpellier Cedex 5, France. \\ ${ }^{2}$ Institut für Theoretische Physik, Universität Karlsruhe, \\ D-76128 Karlsruhe, FR Germany.
}

\begin{abstract}
We analyze the coupling of CP-even and CP-odd Higgs bosons to a photon and a Z boson in extensions of the Standard Model. In particular, we study in detail the effect of charged Higgs bosons in two-Higgs doublet models, and the contribution of SUSY particle loops in the minimal supersymmetric extension of the Standard Model. The Higgs $-\gamma Z$ coupling can be measured in the decay $Z \rightarrow \gamma+$ Higgs at $e^{+} e^{-}$ colliders running on the $Z$ resonance, or in the reverse process Higgs $\rightarrow Z \gamma$ with the Higgs boson produced at LHC. We show that a measurement of this coupling with a precision at the percent level, which could be the case at future $e^{+} e^{-}$colliders, would allow to distinguish between the lightest SUSY and standard Higgs bosons in large areas of the parameter space.
\end{abstract}




\section{Introduction}

The study of the electroweak symmetry breaking mechanism [1] is one of the most important goals of present and future high-energy colliders. Once the first evidence for Higgs particles is established, it will be crucial to ascertain the underlying dynamics of the Higgs sector. This can be achieved by measuring the couplings of the Higgs particles to the other fundamental particles: in the Standard Model (SM), fermions and gauge bosons acquire masses through the interaction with the Higgs field and the size of their couplings are set by the masses. This is a fundamental prediction of the Higgs mechanism which has to be tested experimentally.

Among these Higgs couplings, the couplings to photons are interesting in many respects. First, the interaction of the Higgs particle with photons does not occur at the tree-level since the photon is massless. The Higgs-photon coupling is therefore induced by loops of heavy charged particles. In the SM, this occurs via $W$ boson and heavy fermion triangle loops. Since the couplings of these particles to the Higgs boson grow with the mass, they balance the decrease of the triangle amplitude with increasing loop mass, and the particles do not decouple even for very large masses. Therefore these processes can serve to count the number of heavy charged particles which couple to the Higgs boson.

In supersymmetric (SUSY) theories, the Higgs sector must be extended to contain (at least) two doublets of scalar fields, leading to the existence of five Higgs particles: two $\mathrm{CP}$-even Higgs bosons $h$ and $H$, a $\mathrm{CP}$-odd Higgs boson $A$ as well as two charged Higgs particles $H^{ \pm}$[1]. Depending on the value of $\tan \beta$ [the ratio of the two vacuum expectation values of the Higgs fields], the lightest SUSY Higgs boson $h$ is constrained to be lighter than $M_{h} \lesssim 80-130 \mathrm{GeV}$ [2] in the minimal version. For small values $\tan \beta \sim 1.5$, which are favored by Yukawa coupling unification [3], the Higgs boson has a mass which does not exceed $M_{h} \sim 80 \mathrm{GeV}$, and therefore can be produced at LEP2 [4]. In the decoupling regime $M_{A} \sim M_{H} \sim M_{H^{ \pm}} \gg M_{Z}$ [5], the $h$ boson has practically the same properties as the SM Higgs particle; the MSSM and SM Higgs sectors then look almost the same, and are very difficult to be distinguished. However, additional contributions to the Higgs-photon couplings will be induced by loops with charged Higgs bosons, charginos and sfermions. Since the SUSY particles do not couple to the Higgs boson proportionally to their masses, their contributions decouple for high masses. If, however, some of these particles are not too heavy their contributions can be large enough to allow for a discrimination between the lightest SUSY and standard Higgs bosons even in the decoupling regime.

The Higgs coupling to two photons has received much attention in the literature $[6,7]$. In the SM, the coupling of the Higgs boson $H^{0}$ to a photon and a $Z$ boson has been discussed in Ref. [8]. If $M_{Z}<M_{H^{0}} \lesssim 130 \mathrm{GeV}$, the $H^{0} Z \gamma$ vertex can be measured in the decay process $H^{0} \rightarrow Z \gamma$. At LHC the production rate for light Higgs bosons is very large, $\sigma\left(g g \rightarrow H^{0}\right) \sim 100 \mathrm{pb}[9]$, and despite of the small branching ratio 
$\operatorname{BR}\left(H^{0} \rightarrow Z \gamma\right) \sim 10^{-3}$, one would still have $\mathcal{O}\left(10^{3}\right) Z \gamma$ events if the luminosity is high enough, $\mathcal{L} \sim 10^{34} \mathrm{~cm}^{-2} \mathrm{~s}^{-1}$. The coupling could be therefore measured if the background can be reduced to a manageable level and if the theoretical prediction for the cross section is well under control [10].

If $M_{H^{0}}<M_{Z}$, the $H^{0} Z \gamma$ coupling can be measured in the reverse decay $Z \rightarrow H^{0} \gamma$. At LEP and SLC, the rates are however rather small, $\operatorname{BR}\left(Z \rightarrow H^{0} \gamma\right) \lesssim 10^{-5}$, leading to only a few events. However, at future $e^{+} e^{-}$colliders [11] with the expected integrated luminosities of $\int \mathcal{L} \sim 50 \mathrm{fb}^{-1}$, running a few weeks on the $Z$ resonance would allow to obtain a very large sample of $Z \rightarrow H^{0} \gamma$ events. A very precise measurement of the $H^{0} Z_{\gamma}$ coupling would be possible in this case. If the Higgs boson is discovered at LEP2, one would then use the Next Linear $e^{+} e^{-}$Collider to measure the $H^{0} Z \gamma$ coupling and check whether the Higgs boson is SM-like or not. This measurement would be then equivalent to measuring the $H^{0} \gamma \gamma$ coupling at high-energy $\gamma \gamma$ colliders [12].

In supersymmetric theories, the couplings of the light $\mathrm{CP}$-even and $\mathrm{CP}$-odd Higgs bosons to $Z \gamma$ have been studied some time ago in Ref. [13] (see also [14]). However, these analyses need to be updated for several reasons: (i) the radiative corrections in the MSSM Higgs sector turned out to be very large [2], and therefore must be included; (ii) the Higgs couplings to top squarks can be strongly enhanced if squark mixing is included and this might induce large contributions to the Higgs $-Z \gamma$ coupling, a possibility which has been overlooked; (iii) stronger experimental bounds on the masses of charginos and sfermions are now available [15], eliminating a large part of the SUSY parameter space where contributions from these particles are large; (iv) finally, a fully analytic expression for the contributions of charginos and top squarks with different masses is still lacking.

In this paper, we address all the previous points. Furthermore, we discuss in some details the possibility of using the Higgs $-Z \gamma$ couplings to discriminate between the Standard Model and its extensions. In particular, we analyze to what extent one can use the SUSY loop contributions to distinguish between the standard and light SUSY Higgs boson in the decoupling limit where all other Higgs [and SUSY] particles are too heavy to be produced directly; we will show that this is indeed possible if the coupling can be measured at the percent level. We also show that in a general two Higgs-doublet model, the contributions of charged Higgs bosons do not necessarily decouple from the Higgs- $Z \gamma$ amplitude for large $H^{ \pm}$masses, contrary to the SUSY case.

The paper is organized as follows. In the next section, for completeness and to set up the notation, we discuss the $H^{0} Z \gamma$ coupling in the Standard Model. In section 3, we analyse the Higgs $-Z \gamma$ coupling in the two Higgs-doublet extension of the model. In section 4 , we discuss the various loop contributions of the SUSY particles to the coupling in the MSSM, paying special attention to the small $\tan \beta$ region and the decoupling limit. Our conclusions are given in section 5 . 


\section{The Higgs $-\mathrm{Z}-$ photon coupling in the $\mathrm{SM}$}

For the sake of completeness and to fix our notation, we first discuss the Higgs- $Z \gamma$ coupling in the Standard Model. The $H^{0} Z \gamma$ vertex is mediated by $W$ boson and heavy quark [in practice only top and bottom quark] loops; Fig. 1a. It can be decomposed into the following tensorial structure:

$V\left[Z^{\mu}\left(p_{1}\right), \gamma^{\nu}\left(p_{2}\right), H^{0}\left(p_{3}\right)\right]=F_{0} p_{2}^{\mu} p_{1}^{\nu}+F_{1} g^{\mu \nu}+F_{2} p_{2}^{\mu} p_{2}^{\nu}+F_{3} p_{1}^{\mu} p_{2}^{\nu}+F_{4} p_{1}^{\mu} p_{1}^{\nu}+F_{5} \epsilon^{\mu \nu \alpha \beta} p_{1 \alpha} p_{2 \beta}$

For on-shell particles, only the form factor $F_{0}$ contributes to the decay widths; normalized to $e^{3} /\left(s_{W} M_{W}\right)$ with $s_{W}^{2}=1-c_{W}^{2} \equiv \sin ^{2} \theta_{W}$, it is given by

$$
F_{0}=A_{W}+A_{f} \equiv M_{Z}^{2}\left[\cot \theta_{W} F_{W}+\sum_{f} 2 Q_{f} N_{c} \frac{m_{f}^{2}}{M_{Z}^{2}} \frac{I_{3}^{f}-2 s_{W}^{2} Q_{f}}{s_{W} c_{W}} F_{f}\right]
$$

with $Q_{f}, I_{3}^{f}$ and $m_{f}$ the charge, the weak isospin and the mass of the fermion $f ; N_{c}=1$ for leptons and $N_{c}=3$ for quarks.

In the following we discuss the various contributions in the case of the decay $Z \rightarrow H^{0} \gamma$; the amplitudes for the reverse decay can be simply obtained by crossing. In terms of the Passarino-Veltman three-point scalar functions [16]

$$
C_{0,2}\left(m^{2}\right) \equiv C_{0,2}\left(M_{Z}^{2}, 0, M_{H^{0}}^{2} ; m, m, m\right)
$$

the fermionic and $W$ contributions ${ }^{1}$ are found to be

$$
\begin{aligned}
F_{f} & =C_{0}\left(m_{f}^{2}\right)+4 C_{2}\left(m_{f}^{2}\right) \\
F_{W} & =2\left[\frac{M_{H^{0}}^{2}}{M_{W}^{2}}\left(1-2 c_{W}^{2}\right)+2\left(1-6 c_{W}^{2}\right)\right] C_{2}\left(M_{W}^{2}\right)+4\left(1-4 c_{W}^{2}\right) C_{0}\left(M_{W}^{2}\right)
\end{aligned}
$$

$C_{0}$ is the scalar integral, and $C_{2}$ is a short-hand notation for $C_{2} \equiv C_{11}+C_{23}$ where the expressions of the $C_{i j}$ can be found in [17]. Since there is only one mass running in the loops, the functions $C_{0}\left(m^{2}\right)$ and $C_{2}\left(m^{2}\right)$ have a rather simple form; in terms of the scaled variables $\tau_{Z}=4 m^{2} / M_{Z}^{2}$ and $\tau_{H}=4 m^{2} / M_{H^{0}}^{2}$, they are given by the known expressions [1]

$$
\begin{aligned}
4 m^{2} C_{2}\left(m^{2}\right) & =\frac{\tau_{Z} \tau_{H}}{2\left(\tau_{Z}-\tau_{H}\right)}+\frac{\tau_{Z} \tau_{H}^{2}}{2\left(\tau_{Z}-\tau_{H}\right)^{2}}\left(\tau_{Z}\left[f\left(\tau_{Z}\right)-f\left(\tau_{H}\right)\right]+2\left[g\left(\tau_{Z}\right)-g\left(\tau_{H}\right)\right]\right) \\
4 m^{2} C_{0}\left(m^{2}\right) & =-\frac{2 \tau_{Z} \tau_{H}}{\tau_{Z}-\tau_{H}}\left[f\left(\tau_{Z}\right)-f\left(\tau_{H}\right)\right]
\end{aligned}
$$

with the functions $f$ and $g$ defined by [1]

$$
f(\tau)= \begin{cases}\arcsin ^{2} \sqrt{1 / \tau} & \tau \geq 1 \\ -\frac{1}{4}\left[\log \frac{1+\sqrt{1-\tau}}{1-\sqrt{1-\tau}}-i \pi\right]^{2} & \tau<1\end{cases}
$$

\footnotetext{
${ }^{1}$ We have calculated the amplitudes in the Feynman gauge; however, the results for the fermion and $W$ loops are separately gauge invariant if all external particles are on-shell.
} 


$$
g(\tau)= \begin{cases}\sqrt{\tau-1} \arcsin \sqrt{1 / \tau} & \tau \geq 1 \\ \frac{1}{2} \sqrt{1-\tau}\left[\log \frac{1+\sqrt{1-\tau}}{1-\sqrt{1-\tau}}-i \pi\right] & \tau<1\end{cases}
$$

The $W$ boson and top quark form factors $A_{W}$ and $A_{f}$ are shown in Fig. 2a as a function of the Higgs boson mass. The $b$ quark contribution as well as the contributions of the other fermions are much smaller, due to the small masses. In the range of interest $70 \mathrm{GeV}$ $\lesssim M_{H^{0}} \lesssim 130 \mathrm{GeV}$, the $W$ contribution is by far dominant, being one order of magnitude larger than the top quark contribution; the two amplitudes interfere destructively. The QCD corrections to the top quark loop are small, being of $\mathcal{O}\left(\alpha_{s} / \pi\right)$ [18].

The decay rate for the process $Z \rightarrow H^{0} \gamma$ reads in terms of $F_{0}$ :

$$
\Gamma\left(Z \rightarrow H^{0} \gamma\right)=\frac{\alpha G_{F}^{2} M_{W}^{2} s_{W}^{2}}{192 \pi^{4}} M_{Z}^{3}\left(1-\frac{M_{H^{0}}^{2}}{M_{Z}^{2}}\right)^{3}\left|F_{0}\right|^{2}
$$

This rate, normalized to the total decay width $\Gamma_{Z} \simeq 2.5 \mathrm{GeV}$, is displayed in Fig. 2b. The branching ratio varies from $\sim 10^{-6}$ for masses $M_{H^{0}} \sim 50 \mathrm{GeV}$ [which are ruled out in the SM, but are still possible [15] in extensions of the model] to $\sim 10^{-7}$ for $M_{H^{0}} \sim 80 \mathrm{GeV}$ which can be probed at LEP2 [4]. This means that only a few events can be produced at LEP1 with the present sample of $\mathcal{O}\left(10^{7}\right) Z$ bosons collected by all four collaborations. However, a future collider with the expected yearly integrated luminosity of $\int \mathcal{L} \sim 100$ $\mathrm{fb}^{-1}$, will be able to produce $\mathcal{O}\left(10^{10}\right) Z$ bosons per year; this translates into $\mathcal{O}\left(10^{3}\right)$ $Z \rightarrow H^{0} \gamma$ events for Higgs boson masses not too close to the $M_{H^{0}} \sim M_{Z}$ threshold. Since the signal is very clean [the photon being monochromatic and the decay products of the Higgs boson, $H^{0} \rightarrow b \bar{b}$, being efficiently tagged with micro-vertex detectors], one could measure the $H^{0} Z \gamma$ coupling with a statistical precision of a few percent allowing for a stringent test of the $H^{0} Z \gamma$ coupling. One would therefore check whether the coupling is SM-like, and measure with a good precision the $H^{0} W W$ and $H^{0} t \bar{t}$ coupling.

If $M_{H^{0}}>M_{Z}$, the decay rate for the reverse process $H^{0} \rightarrow Z \gamma$ reads

$$
\Gamma\left(H^{0} \rightarrow Z \gamma\right)=\frac{\alpha G_{F}^{2} M_{W}^{2} s_{W}^{2}}{64 \pi^{4}} M_{H^{0}}^{3}\left(1-\frac{M_{Z}^{2}}{M_{H^{0}}^{2}}\right)^{3}\left|F_{0}\right|^{2}
$$

with $F_{0}$ given by eq. (2). The branching ratio when the $Z$ boson is decaying into electron and muon pairs is also shown in Fig. 2a [with $\operatorname{BR}\left(Z \rightarrow e^{+} e^{-}+\mu^{+} \mu^{-}\right) \sim 6 \%$; other decays of the $Z$ bosons will be rather difficult to extract from the background at the LHC]. In the mass range $M_{H^{0}} \sim 120 \mathrm{GeV}$, the branching ratio is of the order of $10^{-4}$. With the $\mathcal{O}\left(10^{6}\right)$ Higgs bosons produced at LHC in the main production mechanism $g g \rightarrow H^{0}$ with an expected yearly luminosity of $\int \mathcal{L} \sim 100 \mathrm{fb}^{-1}$, a few hundred $H^{0} \rightarrow Z \gamma$ events could be collected in a few years of running, if background problems can be reduced to a manageable level. This rises the hope to measure the $H^{0} Z \gamma$ coupling once the Higgs boson is observed in the $H^{0} \rightarrow \gamma \gamma$ mode for instance. 


\section{The coupling in Two-Higgs Doublet Models}

In a Two Higgs-Doublet Model (THDM), the most general Higgs potential compatible with gauge invariance, the correct breaking of the $\mathrm{SU}(2) \times \mathrm{U}(1)$ symmetry and $\mathrm{CP}$ conservation is given by [1]

$$
\begin{aligned}
V= & \lambda_{1}\left(\left|\phi_{1}\right|^{2}-v_{1}^{2}\right)^{2}+\lambda_{2}\left(\left|\phi_{2}\right|^{2}-v_{2}^{2}\right)^{2}+\lambda_{3}\left[\left(\left|\phi_{1}\right|^{2}-v_{1}^{2}\right)+\left(\left|\phi_{2}\right|^{2}-v_{2}^{2}\right)\right]^{2} \\
& +\lambda_{4}\left[\left|\phi_{1}\right|^{2}\left|\phi_{2}\right|^{2}-\left|\phi_{1}^{\dagger} \phi_{2}\right|^{2}\right]+\lambda_{5}\left[\operatorname{Re}\left(\phi_{1}^{\dagger} \phi_{2}\right)-v_{1} v_{2}\right]^{2}+\lambda_{6}\left[\operatorname{Im}\left(\phi_{1}^{\dagger} \phi_{2}\right)\right]^{2}
\end{aligned}
$$

with $\phi_{1}, \phi_{2}$ the two Higgs-doublet fields and $v_{1}, v_{2}$ their vacuum expectation values. We have also assumed that the discrete symmetry $\phi_{1} \rightarrow-\phi_{1}$ is only broken softly; an additional term, $\lambda_{7}\left[\operatorname{Re}\left(\phi_{1}^{\dagger} \phi_{2}\right)-v_{1} v_{2}\right] \operatorname{Im}\left(\phi_{1}^{\dagger} \phi_{2}\right)$, can be eliminated by redefining the phases of the scalar fields [1]. Parameterizing the Higgs doublets by

$$
\phi_{1}=\left(\begin{array}{c}
\phi_{1}^{+} \\
v_{1}+\eta_{1}+i \chi_{1}
\end{array}\right), \quad \phi_{2}=\left(\begin{array}{c}
\phi_{2}^{+} \\
v_{2}+\eta_{2}+i \chi_{2}
\end{array}\right)
$$

one obtains for the mass terms in the $\mathrm{CP}$-even Higgs sector

$$
\left(\eta_{1}, \eta_{2}\right)\left(\begin{array}{cc}
4\left(\lambda_{1}+\lambda_{3}\right) v_{1}^{2}+\lambda_{5} v_{2}^{2} & \left(4 \lambda_{3}+\lambda_{5}\right) v_{1} v_{2} \\
\left(4 \lambda_{3}+\lambda_{5}\right) v_{1} v_{2} & 4\left(\lambda_{2}+\lambda_{3}\right) v_{2}^{2}+\lambda_{5} v_{1}^{2}
\end{array}\right)\left(\begin{array}{l}
\eta_{1} \\
\eta_{2}
\end{array}\right)
$$

while in the $\mathrm{CP}$-odd and charged Higgs sectors, one has

$$
\lambda_{6}\left(\chi_{1}, \chi_{2}\right)\left(\begin{array}{cc}
v_{2}^{2} & -v_{1} v_{2} \\
-v_{1} v_{2} & v_{1}^{2}
\end{array}\right)\left(\begin{array}{l}
\chi_{1} \\
\chi_{2}
\end{array}\right), \lambda_{4}\left(\phi_{1}^{-}, \phi_{2}^{-}\right)\left(\begin{array}{cc}
v_{2}^{2} & -v_{1} v_{2} \\
-v_{1} v_{2} & v_{1}^{2}
\end{array}\right)\left(\begin{array}{l}
\phi_{1}^{+} \\
\phi_{2}^{+}
\end{array}\right)
$$

Diagonalizing the mass matrices, one obtains the physical masses

$$
\begin{gathered}
M_{H, h}^{2}=\frac{1}{2}\left[\mathcal{M}_{11}+\mathcal{M}_{22} \pm \sqrt{\left(\mathcal{M}_{11}-\mathcal{M}_{22}\right)^{2}+4 \mathcal{M}_{12}^{2}}\right] \\
M_{A}^{2}=\lambda_{6} v^{2} \text { and } M_{H^{ \pm}}^{2}=\lambda_{4} v^{2}
\end{gathered}
$$

with $v^{2} \equiv v_{1}^{2}+v_{2}^{2}$ and $\mathcal{M}$ the mass matrix of eq. (12). The mixing angle $\alpha$ in the $\mathrm{CP}$-even Higgs sector is obtained from

$$
\cos 2 \alpha=\frac{\mathcal{M}_{11}-\mathcal{M}_{22}}{\sqrt{\left(\mathcal{M}_{11}-\mathcal{M}_{22}\right)^{2}+4 \mathcal{M}_{12}^{2}}}, \sin 2 \alpha=\frac{2 \mathcal{M}_{12}}{\sqrt{\left(\mathcal{M}_{11}-\mathcal{M}_{22}\right)^{2}+4 \mathcal{M}_{12}^{2}}}
$$

Inverting these relations, one obtains the $\lambda$ 's in terms of the Higgs masses, and $\alpha, \beta$ :

$$
\begin{aligned}
& \lambda_{1}=\frac{1}{4 \cos ^{2} \beta v^{2}}\left(\cos ^{2} \alpha M_{H}^{2}+\sin ^{2} \alpha M_{h}^{2}\right)-\frac{\sin 2 \alpha}{\sin 2 \beta} \frac{M_{H}^{2}-M_{h}^{2}}{4 v^{2}}+\frac{\lambda_{5}}{4}\left(1-\frac{\sin ^{2} \beta}{\cos ^{2} \beta}\right), \\
& \lambda_{2}=\frac{1}{4 \sin ^{2} \beta v^{2}}\left(\sin ^{2} \alpha M_{H}^{2}+\cos ^{2} \alpha M_{h}^{2}\right)-\frac{\sin 2 \alpha}{\sin 2 \beta} \frac{M_{H}^{2}-M_{h}^{2}}{4 v^{2}}+\frac{\lambda_{5}}{4}\left(1-\frac{\cos ^{2} \beta}{\sin ^{2} \beta}\right), \\
& \lambda_{3}=\frac{\sin 2 \alpha}{\sin 2 \beta} \frac{M_{H}^{2}-M_{h}^{2}}{4 v^{2}}-\frac{\lambda_{5}}{4}, \lambda_{4}=\frac{M_{H^{ \pm}}^{2}}{v^{2}}, \quad \lambda_{6}=\frac{M_{A}^{2}}{v^{2}}
\end{aligned}
$$


As one can see, the parameter $\lambda_{5}$ can not be fixed by the masses and the mixing angles, unless one imposes a strict $\phi_{1} \rightarrow-\phi_{1}$ symmetry resulting in $\lambda_{5}=0$, or by using the SUSY relation $\lambda_{5}=\lambda_{6}=M_{A}^{2} / v^{2}$, as will be discussed later.

In a general THDM, the four masses $M_{h}, M_{H}, M_{A}$ and $M_{H^{ \pm}}$as well as the mixing angles $\alpha$ and $\beta$ are free parameters. The interaction of the Higgs bosons with fermions are model-dependent; here, we will consider the model where one Higgs doublet couples only to up-type quarks, while the other doublet couples only to down-type quarks and charged leptons [the so-called Model II [1] which occurs in SUSY models for instance]. In this case, the couplings of the neutral Higgs boson, collectively denoted by $\Phi$, to fermions and massive gauge bosons are given in Tab. 1, normalized to the SM Higgs couplings. Due to CP invariance, the pseudoscalar $A$ does not couple to $W$ and $Z$ bosons.

\begin{tabular}{|c|c|c|c|}
\hline$\Phi$ & $g_{\Phi \bar{u} u}$ & $g_{\Phi \bar{d} d}$ & $g_{\Phi V V}$ \\
\hline$h$ & $\cos \alpha / \sin \beta$ & $-\sin \alpha / \cos \beta$ & $\sin (\beta-\alpha)$ \\
$H$ & $\sin \alpha / \sin \beta$ & $\cos \alpha / \cos \beta$ & $\cos (\beta-\alpha)$ \\
$A$ & $1 / \tan \beta$ & $\tan \beta$ & 0 \\
\hline
\end{tabular}

Tab. 1 Higgs boson couplings to fermions and gauge bosons in the THDM compared to the SM Higgs couplings.

For the $\mathrm{CP}$-even Higgs bosons, the couplings to photons and $Z$ bosons receive contributions from $W$ and top/bottom quark loops as well as contributions from charged Higgs boson loops (Fig. 1b). The structure of the vertex is again given by eq. (1), and only the form factor $F_{0}$ contributes for the decay. It is given by $[\phi \equiv h, H]$

$$
\begin{aligned}
F_{0}=M_{Z}^{2}[ & \cot \theta_{W} g_{\phi V V} F_{W}+\cot \theta_{W} g_{\phi H^{+} H^{-}} F_{H^{ \pm}} \\
& \left.+\sum_{f} 2 Q_{f} N_{c} \frac{m_{f}^{2}}{M_{Z}^{2}} \frac{I_{3}^{f}-2 s_{W}^{2} Q_{f}}{s_{W} c_{W}} g_{\phi f f} F_{f}\right] .
\end{aligned}
$$

The functions $F_{W}$ and $F_{f}$ are the same as previously, while the function $F_{H^{ \pm}}$for the charged Higgs contribution reads in term of the $C_{2}$ function defined previously (see also [19]):

$$
F_{H^{ \pm}}=4 C_{2}\left(M_{H^{ \pm}}^{2}\right) \text {. }
$$

The couplings $g_{\phi V V}$ and $g_{\phi f f}$ can be taken from Tab. 1, while the couplings of the CPeven neutral Higgs bosons to charged Higgs bosons in the THDM [using a normalization similar to the one for the $W$ boson and the fermions] are found to be

$$
\begin{aligned}
g_{h H^{+} H^{-}} & =\frac{M_{h}^{2}-\lambda_{5} v^{2}}{M_{W}^{2}} \frac{\cos (\beta+\alpha)}{\sin 2 \beta}+\frac{2 M_{H^{ \pm}}^{2}-M_{h}^{2}}{2 M_{W}^{2}} \sin (\beta-\alpha) \\
g_{H H^{+} H^{-}} & =\frac{M_{H}^{2}-\lambda_{5} v^{2}}{M_{W}^{2}} \frac{\sin (\beta+\alpha)}{\sin 2 \beta}+\frac{2 M_{H^{ \pm}}^{2}-M_{H}^{2}}{2 M_{W}^{2}} \cos (\beta-\alpha) .
\end{aligned}
$$


In the limit of very heavy $H^{ \pm}$bosons, the $C_{2}\left(M_{H^{ \pm}}^{2}\right)$ function reduces to

$$
C_{2}\left(M_{H^{ \pm}}^{2}\right) \rightarrow 1 /\left(24 M_{H^{ \pm}}^{2}\right),
$$

while the coupling of the $h$ boson [that we assume to be the lighter CP-even Higgs state] to the charged Higgs boson approaches the limit

$$
g_{h H^{+} H^{-}} \rightarrow \frac{M_{H^{ \pm}}^{2}}{M_{W}^{2}} \sin (\beta-\alpha)
$$

assuming that $\lambda_{5} v^{2} \ll M_{H^{ \pm}}^{2}$. This leads to a final contribution which is proportional to $\sin ^{2}(\beta-\alpha) \equiv g_{h V V}^{2}$. Therefore, the charged Higgs contribution to the $h Z \gamma$ coupling in a general THDM does not decouple, contrary to the case of SUSY models as will be discussed later. However, the $H^{ \pm}$contribution is suppressed by the large factor $1 / 24$ and compared to the $W$ boson loop [which in a THDM, is also damped by the factor $\sin (\beta-\alpha)$ compared to the SM case], it is two orders of magnitude smaller. The decay widths $\Gamma(Z \rightarrow h \gamma)$ or $\Gamma(h \rightarrow Z \gamma)$, given by eqs. (8-9) with $M_{H^{0}} \rightarrow M_{h, H}$, will therefore be hardly sensitive to this loop effect.

We now turn to the case of the pseudoscalar Higgs boson $A$. Due to CP-invariance, the $A Z \gamma$ coupling is induced only by fermionic loops, since $A$ does not couple to $W$ and $H^{ \pm}$bosons. Its tensorial structure is given by the same expression as in eq. (1), but here only the form factor $F_{5}$ contributes. The decay widths $\Gamma(Z \rightarrow A \gamma)$ or $\Gamma(A \rightarrow Z \gamma)$ are given by eqs. (8-9) with $M_{H^{0}} \rightarrow M_{A}$ and $F_{0}$ replaced by

$$
F_{5}=-\sum_{f} 2 Q_{f} N_{c} \frac{m_{f}^{2}}{M_{W}^{2}}\left(I_{3}^{f}-2 s_{W}^{2} Q_{f}\right) g_{A f f} C_{0}\left(m_{f}^{2}\right) .
$$

In the general THDM, a numerical analysis is rather complicated [and not very telling] since besides the four masses $M_{h}, M_{H}, M_{A}$ and $M_{H^{ \pm}}$, we have the mixing angles $\alpha$ and $\beta$ as additional parameters, not to mention the parameter $\lambda_{5}$ which is also independent. The Higgs $-Z \gamma$ couplings can vary widely compared to the SM coupling, although the dominant $W$ boson amplitude is always suppressed by the factors $\sin (\beta-\alpha)$ or $\cos (\beta-\alpha)$ in the case of the $\mathrm{CP}$-even bosons or absent in the case of the pseudoscalar $A$. To simplify the discussion, we will use the constraints provided by supersymmetry: in the MSSM, the Higgs sector is described at the tree-level only by two free parameters that we chose to be $\tan \beta$ and the pseudoscalar mass $M_{A}$. The masses of the CP-even Higgs bosons are given by

$$
M_{h, H}^{2}=\frac{1}{2}\left[M_{A}^{2}+M_{Z}^{2} \mp \sqrt{\left(M_{A}^{2}+M_{Z}^{2}\right)^{2}-4 M_{A}^{2} M_{Z}^{2} \cos ^{2} 2 \beta}\right]
$$

while the mass of the charged Higgs boson is simply given by

$$
M_{H^{ \pm}}^{2}=M_{A}^{2}+M_{W}^{2}
$$


The mixing angle $\alpha$ is related to $M_{A}$ and $\tan \beta$ by

$$
\tan 2 \alpha=\tan 2 \beta \frac{M_{A}^{2}+M_{Z}^{2}}{M_{A}^{2}-M_{Z}^{2}} \quad, \quad-\frac{\pi}{2} \leq \alpha \leq 0
$$

However, these relations are affected by large radiative corrections [2] which must be taken into account. We will therefore include the leading radiative correction to the Higgs masses and couplings which grows as $m_{t}^{4}$ and logarithmically with the common squark mass that we fix to $1 \mathrm{TeV}$, unless otherwise stated. In the MSSM, one has $1<\tan \beta<m_{t} / m_{b}$ from GUT restrictions, with the lower $[\tan \beta \sim 1.6]$ and the upper $[\tan \beta \sim 50]$ ranges favored by Yukawa coupling unification [3]. We will mainly focus on the lightest $\mathrm{CP}$-even Higgs boson, for which the maximum allowed value of the mass is about $M_{h}^{\max } \simeq 80 \mathrm{GeV}$ for $\tan \beta \sim 1.6$, and the particle is therefore accessible in $Z$ decays. In the high $\tan \beta$ range, the maximal $h$ mass can reach values $M_{h} \sim 130 \mathrm{GeV}$, and the decay $h \rightarrow Z \gamma$ would be kinematically possible.

The $W$ boson amplitude $A_{W}=\cot \theta_{W} g_{h V V} F_{W}$ is shown in Fig. 3a as a function of $M_{h}$ for the three values $\tan \beta=1.6,5$ and 50. For low $h$ masses, $A_{W}$ is suppressed compared to the SM value, the suppression being more effective with increasing $\tan \beta$; in fact, for $\tan \beta \sim 50$ the $W$ contribution almost vanishes. With increasing $h$ mass, $A_{W}$ approaches the SM value which is reached for $M_{h}=M_{h}^{\max }$. The sum of the top and bottom loop contributions is also displayed in Fig. 3a. Except when $M_{h} \sim M_{h}^{\max }$, where the the form factor $A_{f}$ becomes SM-like, the $t$ contribution is suppressed by a factor $g_{h t t} \sim 1 / \tan \beta$, while the $b$ contribution is enhanced by the factor $g_{h b b} \sim \tan \beta$. Therefore, the $t$ contribution is dominant for low $\tan \beta$, while for large $\tan \beta$ values the $b$ contribution [which has opposite sign compared to the SM case] is strongly enhanced and becomes dominant.

The contribution of the charged Higgs boson loop $A_{H^{ \pm}}=\cot \theta_{W} g_{h H^{+} H^{-}} F_{H^{ \pm}}$is shown in Fig. 3b as a function of $M_{H^{ \pm}}$for the values $\tan \beta=1.6,5$ and 50. The behavior can be understood by recalling the expression of the $g_{h H^{+} H^{-}}$coupling in the MSSM

$$
g_{h H^{+} H^{-}}=\sin (\beta-\alpha)+\frac{\cos 2 \beta \sin (\beta+\alpha)}{2 c_{W}^{2}},
$$

in which the radiative correction must also be included; see for instance Ref. [7]. For small $M_{H^{ \pm}}$implying small $M_{h}$, the coupling is strongly suppressed for large $\tan \beta$ values and the contribution $A_{H^{ \pm}}$is small. For $\tan \beta \sim 1$, the suppression is rather mild and the $H^{ \pm}$ contribution can be large, reaching a few percent of the $W$ contribution for $M_{H^{ \pm}} \sim 100$ GeV. Contrary to the THDM, $A_{H^{ \pm}}$decreases with increasing $M_{H^{ \pm}}$since in the MSSM, the $g_{\mathrm{hH}^{+} H^{-}}$coupling does not scale like the charged Higgs mass, and the contribution is damped by a factor $1 / M_{H^{ \pm}}^{2}$. The charged Higgs boson therefore yields small contributions to the $h Z \gamma$ coupling and decouples from the vertex for high masses. 


\section{The Higgs $-\mathrm{Z}-$ Photon coupling in the MSSM}

In the Minimal Supersymmetric extension of the Standard Model, the couplings of the $\mathrm{CP}$-even Higgs bosons to the photon and the $Z$ boson receive, as in the Two HiggsDoublet Model, contributions from $W$ bosons, top+bottom quarks and charged Higgs bosons. Extra contributions also come from charged supersymmetric particles: sleptons, squarks and charginos (Fig. 1c). The decay widths are again given by eqs. (8-9) with $M_{H^{0}} \rightarrow M_{\phi}$, and the form factor $F_{0}$ reads:

$$
\begin{aligned}
F_{0}=M_{Z}^{2}[ & \cot \theta_{W} g_{\phi V V} F_{W}+\sum_{f} 2 Q_{f} N_{c} \frac{m_{f}^{2}}{M_{Z}^{2}} \frac{I_{3}^{f}-2 s_{W}^{2} Q_{f}}{s_{W} c_{W}} g_{\phi f f} F_{f} \\
& \left.+\cot \theta_{W} g_{\phi H^{+} H^{-}} F_{H^{ \pm}}+\cot \theta_{W} F_{\chi^{+}}+\sum_{\tilde{f}} N_{c} Q_{\tilde{f}} F_{\tilde{f}}\right] .
\end{aligned}
$$

The amplitudes from the fermions, $W$ and $H^{ \pm}$bosons are, as in the previous section, given by eqs. (4) and (18), while the chargino contribution reads

$$
F_{\chi^{+}}=\sum_{j, k=1,2} \frac{m_{\chi_{j}^{+}}}{M_{W}} f\left(m_{\chi_{j}^{+}}, m_{\chi_{k}^{+}}, m_{\chi_{k}^{+}}\right) \sum_{m, n=L, R} g_{Z \chi_{j}^{+} \chi_{k}^{-}}^{m} g_{\phi \chi_{k}^{+} \chi_{j}^{-}}^{n}
$$

The couplings of charginos to the $Z$ bosons are given by

$$
g_{Z \chi_{j}^{+} \chi_{k}^{-}}^{L}=-\left(V_{i 1} V_{j 1}^{*}+\frac{1}{2} V_{i 2} V_{j 2}^{*}-\delta_{i j} s_{W}^{2}\right), \quad g_{Z \chi_{j}^{+} \chi_{k}^{-}}^{R}=-\left(U_{i 1} U_{j 1}^{*}+\frac{1}{2} U_{i 2} U_{j 2}^{*}-\delta_{i j} s_{W}^{2}\right)
$$

while the couplings to the higgs bosons read

$$
g_{\phi \chi_{i}^{+} \chi_{j}^{-}}^{L}=Q_{j i}^{*} c_{\phi}-S_{j i}^{*} d_{\phi}, g_{\phi \chi_{i}^{+} \chi_{j}^{-}}^{R}=Q_{i j} c_{\phi}-S_{i j} d_{\phi}
$$

with $c_{h} / d_{h}=\sin \alpha / \cos \alpha$ and $c_{H} / d_{H}=-\cos \alpha / \sin \alpha$. The elements $Q_{i j} / S_{i j}$, as well as the matrices $V$ and $U$ which diagonalize the chargino mass matrix can be found in [20].

The function $f$ entering the chargino form-factor is given by

$$
\begin{gathered}
f\left(m_{1}, m_{2}, m_{2}\right)=-2\left[C_{0}\left(m_{1}, m_{2}, m_{2}\right)+C_{1}\left(m_{1}, m_{2}, m_{2}\right)+2 C_{2}\left(m_{1}, m_{2}, m_{2}\right)\right. \\
\left.+2 C_{2}\left(m_{2}, m_{1}, m_{1}\right)-C_{1}\left(m_{2}, m_{1}, m_{1}\right)\right]
\end{gathered}
$$

where $C_{1}$ and $C_{2}$ have now a more complicated structure since there are two particles with different masses inside the loop. In terms of the scalar Passarino-Veltman functions $A_{0}, B_{0}$ and $C_{0}$ one has

$$
\begin{aligned}
C_{1}\left(m_{1}, m_{2}, m_{2}\right) & \equiv C_{11}\left(M_{Z}^{2}, 0, M_{h}^{2} ; m_{1}, m_{2}, m_{2}\right) \\
& =\frac{B_{0}\left(M_{h}^{2} ; m_{1}, m_{2}\right)-B_{0}\left(M_{Z}^{2} ; m_{1}, m_{2}\right)}{M_{Z}^{2}-M_{h}^{2}}-C_{0}\left(M_{Z}^{2}, 0, M_{h}^{2} ; m_{1}, m_{2}, m_{2}\right)
\end{aligned}
$$




$$
\begin{aligned}
C_{2}\left(m_{1}, m_{2}, m_{2}\right) \equiv & C_{12}\left(M_{Z}^{2}, 0, M_{h}^{2} ; m_{1}, m_{2}, m_{2}\right)+C_{23}\left(M_{Z}^{2}, 0, M_{h}^{2} ; m_{1}, m_{2}, m_{2}\right) \\
= & \frac{m_{1}^{2}-m_{2}^{2}-M_{Z}^{2}}{2\left(M_{Z}^{2}-M_{h}^{2}\right)^{2}}\left[B_{0}\left(M_{h}^{2} ; m_{1}, m_{2}\right)-B_{0}\left(M_{Z}^{2} ; m_{1}, m_{2}\right)\right] \\
& +\frac{1}{2\left(M_{Z}^{2}-M_{h}^{2}\right) M_{h}^{2}}\left[M_{h}^{2}+2 m_{2}^{2} M_{h}^{2} C_{0}\left(M_{Z}^{2}, 0, M_{h}^{2} ; m_{1}, m_{2}, m_{2}\right)\right. \\
& \left.+\left(m_{2}^{2}-m_{1}^{2}\right) B_{0}\left(M_{h}^{2} ; m_{1}, m_{2}\right)+A_{0}\left(m_{1}\right)-A_{0}\left(m_{2}\right)\right] .
\end{aligned}
$$

The expressions of the scalar one-, two- and three- point functions $A_{0}, B_{0}$ and $C_{0}$ are

$$
\begin{gathered}
A_{0}(m)=m^{2}\left[1-\log \frac{m^{2}}{\mu^{2}}\right] \\
B_{0}\left(p^{2}, m_{1}, m_{2}\right)=2-\log \frac{m_{1} m_{2}}{\mu^{2}}+\frac{m_{1}^{2}-m_{2}^{2}}{p^{2}} \log \frac{m_{2}}{m_{1}} \\
+\frac{\lambda^{1 / 2}\left(p^{2}, m_{1}^{2}, m_{2}^{2}\right)}{p^{2}} \log \frac{m_{1}^{2}+m_{2}^{2}-p^{2}+\lambda^{1 / 2}\left(p^{2}, m_{1}^{2}, m_{2}^{2}\right)}{2 m_{1} m_{2}} \\
C_{0}\left(M_{2}^{2}, 0, M_{1}^{2}, m_{1}, m_{2}, m_{2}\right)= \\
\frac{1}{M_{1}^{2}-M_{2}^{2}} \sum_{i=1}^{2} \sum_{\sigma= \pm 1}(-1)^{i} \operatorname{Li}_{2}\left[\frac{2 M_{i}^{2}}{m_{2}^{2}-m_{1}^{2}+M_{i}^{2}+\sigma \lambda^{1 / 2}\left(M_{i}^{2}, m_{1}^{2}, m_{2}^{2}\right)}\right]
\end{gathered}
$$

$\mu$ is the renormalization scale, and the ultraviolet poles in $A_{0}$ and $B_{0}$ are subtracted since the amplitudes are finite; $\lambda$ is the usual two-body phase space function: $\lambda(x, y, z)=$ $x^{2}+y^{2}+z^{2}-2(x y+x z+y z)$.

Finally, the contribution of the squark and slepton loops to the $h Z \gamma$ couplings reads

$$
F_{\tilde{f}}=-8 \sum_{j, k=1,2} g_{\phi \tilde{f}_{j} \tilde{f}_{k}} g_{Z \tilde{f}_{k} \tilde{f}_{j}} C_{2}\left(m_{\tilde{f}_{j}}, m_{\tilde{f}_{k}}, m_{\tilde{f}_{k}}\right)
$$

with the function $C_{2}$ defined in eq. (30). The squark couplings to the $Z$ boson, including mixing between left-- and right-handed sfermions, are given by

$$
\begin{aligned}
g_{Z \tilde{f}_{1} \tilde{f}_{1}} & =\frac{1}{s_{W} c_{W}}\left[\left(I_{3}^{f}-Q_{f} s_{W}^{2}\right) \cos ^{2} \theta_{f}-Q_{f} s_{W}^{2} \sin ^{2} \theta_{f}\right] \\
g_{Z \tilde{f}_{2} \tilde{f}_{2}} & =\frac{1}{s_{W} c_{W}}\left[-Q_{f} s_{W}^{2} \cos ^{2} \theta_{f}+\left(I_{3}^{f}-Q_{f} s_{W}^{2}\right) \sin ^{2} \theta_{f}\right] \\
g_{Z \tilde{f}_{1} \tilde{f}_{2}} & =\frac{-I_{3}^{f}}{s_{W} c_{W}} \sin \theta_{f} \cos \theta_{f}
\end{aligned}
$$

The mixing is proportional to the fermion mass, and in practice is non-negligible only for the partners of the third generation fermions. 
The couplings of the Higgs bosons to squarks have a more complicated structure because of the squark mixing. In the case of the light $\mathrm{CP}$-even Higgs boson $h$, they read

$$
\begin{aligned}
& g_{h \tilde{f}_{1} \tilde{f}_{1}}=C_{L L}^{h} \cos ^{2} \theta_{f}+C_{R R}^{h} \sin ^{2} \theta_{f}+2 C_{R L}^{h} \cos \theta_{f} \sin \theta_{f} \\
& g_{h \tilde{f}_{2} \tilde{f}_{2}}=C_{R R}^{h} \cos ^{2} \theta_{f}+C_{L L}^{h} \sin ^{2} \theta_{f}-2 C_{R L}^{h} \cos \theta_{f} \sin \theta_{f} \\
& g_{h \tilde{f}_{1} \tilde{f}_{2}}=C_{R L}^{h}\left(\cos ^{2} \theta_{f}-\sin ^{2} \theta_{f}\right)+\left(C_{R R}^{h}-C_{L L}^{h}\right) \cos \theta_{f} \sin \theta_{f}
\end{aligned}
$$

with

$$
\begin{aligned}
C_{L L}^{h} & =\left(I_{3}^{f}-Q_{f} s_{W}^{2}\right) g_{h V V}-\frac{m_{f}^{2}}{M_{Z}^{2}} g_{h f f} \\
C_{R R}^{h} & =\left(Q_{f} s_{W}^{2}\right) g_{h V V}-\frac{m_{f}^{2}}{M_{Z}^{2}} g_{h f f} \\
C_{R L}^{h} & =-\frac{m_{f}}{2 M_{Z}^{2}}\left[A_{f} g_{h f f}-\mu g_{H f f}\right]
\end{aligned}
$$

where $A_{f}$ is the soft-SUSY breaking trilinear term and $\mu$ the Higgs-higgsino mass parameter; the couplings $g_{h f f}$ and $g_{\phi V V}$ are given in Tab. 1 . The heavy CP-even Higgs boson couplings to squarks can be obtained from the previous ones, by performing the substitutions $g_{h \ldots} \leftrightarrow g_{H \ldots}, \sin \alpha \rightarrow \cos \alpha$ and $\cos \alpha \rightarrow \sin \alpha$. Note that in the case of the partners of the light fermions, the mixing angles and the fermion masses can be set to zero, and these couplings simplify considerably.

For the pseudoscalar Higgs boson $A$, only top+bottom quarks and the charginos are contributing to the $A Z_{\gamma}$ amplitude because of $\mathrm{CP}$-invariance. The form factor $F_{0}$ has to be replaced by

$$
F_{5}=-M_{Z}^{2}\left[\sum_{f} 2 Q_{f} N_{c} \frac{m_{f}^{2}}{M_{Z}^{2}} \frac{I_{3}^{f}-2 s_{W}^{2} Q_{f}}{s_{W} c_{W}} g_{A f f} C_{0}\left(m_{f}^{2}\right)-\cot \theta_{W} F_{\chi^{+}}\right]
$$

with the chargino contribution

$$
F_{\chi^{+}}=\sum_{j, k=1,2} \frac{m_{\chi_{j}^{+}}}{M_{Z}} g\left(m_{\chi_{j}^{+}}, m_{\chi_{k}^{+}}, m_{\chi_{k}^{+}}\right)\left(g_{Z \chi_{j}^{+} \chi_{k}^{-}}^{R}+g_{Z \chi_{k}^{+} \chi_{j}^{-}}^{L}\right)\left(g_{A \chi_{j}^{+} \chi_{k}^{-}}^{R}-g_{A \chi_{k}^{+} \chi_{j}^{-}}^{L}\right) .
$$

The $g_{A \chi^{+} \chi^{-}}$couplings are given by

$$
g_{\phi \chi_{i}^{+} \chi_{j}^{-}}^{L}=-Q_{j i}^{*} \sin \beta-S_{j i}^{*} \cos \beta, g_{\phi \chi_{i}^{+} \chi_{j}^{-}}^{R}=Q_{i j} \sin \beta+S_{i j} \cos \beta,
$$

and the new function $g$ reads

$$
g\left(m_{1}, m_{2}, m_{2}\right)=-2\left[C_{0}\left(m_{1}, m_{2}, m_{2}\right)+C_{1}\left(m_{1}, m_{2}, m_{2}\right)+C_{1}\left(m_{2}, m_{1}, m_{1}\right)\right] .
$$

In the MSSM, the CP-even Higgs boson $H$ is always heavier than $M_{Z}$ and therefore only the decay $H \rightarrow Z \gamma$ is kinematically possible. However, even before allowing the 
$Z$ boson to decay into charged leptons, the branching ratio $\mathrm{BR}(H \rightarrow \gamma Z)$ is very small and the process will be very difficult if not impossible to be seen at the LHC. This is also the case of the decay $A \rightarrow Z \gamma$. The process $Z \rightarrow A \gamma$ will be possible if $M_{A} \lesssim 80$ $\mathrm{GeV}$, but then the pseudoscalar Higgs boson can be discovered at LEP2 in the associated production mechanism $e^{+} e^{-} \rightarrow h A[4]$ and its properties can be studied. In the numerical analysis, we therefore focus on the light $\mathrm{CP}$-even Higgs boson $h$ and study in particular the low $\tan \beta$ scenario in which $M_{h} \lesssim 80 \mathrm{GeV}$ and the $h$ boson can be produced at LEP2 in the process $e^{+} e^{-} \rightarrow h Z$. We will pay special attention to the decoupling limit where the $h$ boson mimics the SM Higgs particle and the measurement of the $h Z \gamma$ coupling at future $e^{+} e^{-}$linear colliders running at the $Z$ resonance could help discriminating between the SM and MSSM scenarios.

The contributions of the $W$, SM fermion and charged Higgs boson loops to the $h Z \gamma$ have already been discussed in the THDM with the MSSM constraints. As discussed previously, for large values of $M_{A}$, the $W$ and quark contributions are as in the SM, while the charged Higgs boson decouples and its contribution is negligible. The contributions of the slepton and the scalar partners of the light quarks, neglecting sfermion mixing, are shown in Fig. 4 as functions of the masses and for the three values $\tan \beta=1.6,5$ and 50 with $M_{A}$ fixed to $1 \mathrm{TeV}$. We have summed over all slepton and squark [except stop] contributions, and used common masses $m_{\tilde{l}}$ and $m_{\tilde{q}}$. As in the case of the charged Higgs boson, slepton and squark loop contributions to the $h \rightarrow Z \gamma$ decay width are very small, except when these particles are very close to their allowed mass values [15]. For loop masses above $150 \mathrm{GeV}$, the current experimental bound on squark masses, they do not exceed the level of a few permille of the dominant $W$ contribution and will therefore hardly be detected.

The contribution of the top squark loops to the $h \gamma Z$ vertex depend on the soft SUSYbreaking scalar masses $m_{\tilde{t}_{L}}$ and $m_{\tilde{t}_{R}}$ [which are taken in general to be equal], as well as on the soft-SUSY breaking trilinear term $A_{t}$, the Higgs-higgsino mass parameter $\mu$ and $\tan \beta$. These parameters also determine the masses and mixing angle of the scalar top quarks and their couplings to the Higgs bosons. In the decoupling limit, $M_{A} \gg M_{Z}$ the contribution only depends on the combination $m_{t}^{L R}=A_{t}-\mu / \tan \beta$ and $m_{\tilde{t}_{L}}$ which can be traded against the mass of the lightest top squark $m_{\tilde{t}_{1}}$. In Fig. 5a, we show contour plots in the $\left(m_{t}^{L R}, m_{\tilde{t}_{1}}\right)$ plane for which the contribution $A_{\tilde{t}}$, which includes the amplitudes of both top squarks, is $0.5,0.2$ and 0.1 . For large $M_{A}$, i.e. in the decoupling limit, the amplitude $A_{\tilde{t}}$ is symmetric for positive and negative $m_{t}^{L R}$ values. For large $\left|m_{t}^{L R}\right|$, the contributions are large and negative; for light enough top squarks, $m_{\tilde{t}_{1}} \sim 150 \mathrm{GeV}$, they can reach the value $A_{\tilde{t}} \sim-.5$ for $\left|m_{t}^{L R}\right| \sim 1 \mathrm{TeV}$, i.e. at the level of the top quark contribution. For a given $m_{\tilde{t}_{1}}, A_{\tilde{t}}$ is larger for higher values of $m_{t}^{L R}$, because in this case the coupling $h \tilde{t} \tilde{t} \sim m_{t}^{L R}$ is strongly enhanced. For large $m_{\tilde{t}_{1}}$, the two top squarks will 
have comparable masses and their amplitudes will partly cancel each other, leading to the contour $A_{\tilde{t}}=0$. For small $\left|m_{t}^{L R}\right|$, there is a region around $m_{t}^{L R}=0$ where no solution for $m_{\tilde{t}_{1}}<m_{t}$ is allowed when diagonalizing the mass matrix and this region is already excluded by CDF/D0 data since there one has $m_{\tilde{q}}<150 \mathrm{GeV}$ [15]. The amplitudes in this region are positive and can reach values $A_{\tilde{t}}=0.2$ which decrease with increasing top squark mass as expected.

Fig. 5b shows the contribution $A_{\tilde{t}}$ for $M_{A}=100 \mathrm{GeV}$, i.e. away from the decoupling limit, and $\tan \beta=1.6$ [solid lines] and 50 [dashed lines]. For low $\tan \beta$ values, the symmetry around $m_{t}^{L R}=0$ is lost; the picture is the same as in Fig. $5 \mathrm{a}$ for positive $m_{t}^{L R}$ values, but the contribution $A_{\tilde{t}}$ becomes smaller for negative $m_{t}^{L R}$. For the high $\tan \beta$ scenario, the contribution $A_{\tilde{t}}$ becomes very small.

The contribution of the charginos to the $h Z \gamma$ coupling depends on $\tan \beta$, the gaugino mass parameter $M_{2}$ and the Higgs-higgsino mass parameter $\mu$ [these parameters also fix the chargino masses]. The form factor $A_{\chi}$ is shown in Fig. 6 in the $\left(M_{2}, \mu\right)$ plane for $\tan \beta=1.6$ and $M_{A}=1 \mathrm{TeV}$ and $M_{A}=80 \mathrm{GeV}$. Contours for $\left|A_{\chi}\right|=2,1,0.5$ and 0.2 as well as the region of the parameter space for which the lightest chargino mass is larger than $70 \mathrm{GeV}$ [which approximately corresponds to the current experimental limit] have been drawn. The chargino contributions are rather large close to the $M_{\chi^{+}}=70$ $\mathrm{GeV}$ boundary, reaching values $A_{\chi^{+}} \sim 1$, and become smaller when one moves away from this boundary. However, in a large part of the $\left(M_{2}, \mu\right)$ parameter space, the chargino contribution is larger than $A_{\chi^{+}}=0.2$ and does not strongly depend on $M_{A}$. In fact, in this case, $A_{\chi^{+}}>0.1$ in the entire parameter space $M_{2}, \mu<500 \mathrm{GeV}$ which leads to a deviation of the $h \rightarrow Z \gamma$ coupling by more than one percent.

\section{Summary}

We have analyzed the Higgs- $Z \gamma$ coupling in the Minimal Supersymmetric extension of the Standard Model. We have included the large radiative corrections in the Higgs sector, updated the contributions of the charginos and the top squarks to the coupling, and given fully analytic expressions for these contributions. In Two Higgs-Doublet Models, we have shown that the additional contribution from charged Higgs bosons do not necessarily decouple from the amplitude, if the $H^{ \pm}$mass is large.

We have payed special attention to the case of the MSSM lightest CP-even Higgs boson $h$ in the decoupling limit, where it has almost exactly the properties of the standard Higgs particle. The contributions of the $W$ and top quark loops to the $h Z \gamma$ coupling are the same as in the SM, but additional contributions are induced by chargino and top squark loops. In the low $\tan \beta$ scenario where the $h$ boson is lighter than $\sim 80 \mathrm{GeV}$, these contributions can induce large deviations of the $Z \rightarrow h \gamma$ decay width from the SM value, 
even in the decoupling limit. This is illustrated in Fig. 7, where the deviations due to charginos and $\tilde{t}$ quarks are shown for two values of the masses 100 and $250 \mathrm{GeV}$. As can be seen, in very large areas of the MSSM parameter space, the deviations can exceed the level of several percent.

If future $e^{+} e^{-}$linear colliders could spend a few months running at the $Z$ resonance, a large sample of $Z \rightarrow \gamma+$ Higgs decays could be collected with the expected highluminosities, if the decay is kinematically allowed. In this case, the Higgs $-Z \gamma$ coupling could be measured with a precision at the percent level, allowing a stringent test of the coupling. In the Standard Model, the couplings of the Higgs particle to $W$ bosons and top quarks can be measured with a good precision. In the MSSM, since the contributions of the genuine SUSY particles to the decay width exceed the percent level in large areas of the parameter space, the $h$ boson can be distinguished from the standard Higgs boson even in the decoupling limit. The measurement of the Higgs $-Z \gamma$ coupling in the decay $Z \rightarrow \gamma+$ Higgs will be in this sense, competitive with the measurement of the Higgs couplings to two photons at high-energy $\gamma \gamma$ colliders.

Acknowledgement: Discussions with J. Rosiek are gratefully acknowledged. 


\section{References}

[1] For a review on the Higgs sector of the SM and the MSSM, see J.F. Gunion, H.E. Haber, G.L. Kane and S. Dawson, The Higgs Hunter's Guide, Addison-Wesley, Reading 1990.

[2] Y. Okada, M. Yamaguchi and T. Yanagida, Prog. Theor. Phys. 85 (1991) 1;

H. Haber and R. Hempfling, Phys. Rev. Lett. 66 (1991) 1815;

J. Ellis, G. Ridolfi and F. Zwirner, Phys. Lett. 257B (1991) 83;

R. Barbieri, F. Caravaglios and M. Frigeni, Phys. Lett. 258B (1991) 167;

A. Hoang and R. Hempfling, Phys. Lett. B331 (1994) 99.

[3] V. Barger, M.S. Berger, P. Ohmann, and R.J.N. Phillips, Phys. Lett. B314 (1993) 351 ;

M. Carena, S. Pokorski, and C. E. M. Wagner, Nucl. Phys. B406 (1993) 59.

[4] M. Carena nd P.M. Zerwas [conv.] et al, Report Higgs Physics at LEPII, CERN-9601, G. Altarelli, T. Sjöstrand and F. Zwirner (eds.).

[5] H.E. Haber, CERN-TH/95-109 and SCIPP-95/15, Proceedings, Conference on Physics Beyond the Standard Model IV, Lake Tahoe CA 1994; and Perspectives for Electroweak Interactions in $e^{+} e^{-}$Collisions, Ringberg Castle 1995, ed. B.A. Kniehl

[6] J. Ellis, M.K. Gaillard and D.V. Nanopoulos, Nucl. Phys. B106 (1976) 292;

A. I. Vainshtein, M. Voloshin, V. Zakharov and M. Shifman, Sov. J. Nucl. Phys. 30 (1979) 711;

A. Barroso, J. Pulido and J. C. Romao, Nucl. Phys. B267 (1985) 509;

A. Abbassadi, D. Bowser-Chao, D. Dicus and W. Repko, Phys. Rev. D52 (1995) 3919 .

[7] A. Djouadi, V. Driesen, W. Hollik and J. Rosiek, KA-TP-21-96, hep-ph/9609420, to appear in Nucl. Phys. B.

[8] F. Wilczek, Phys. Rev. Lett. 39 (1977) 1304;

R.N. Cahn, M.S. Chanowitz and N. Fleishon, Phys. Lett. B82 (1979) 113;

L. Bergstrom and G. Hulth, Nucl. Phys. B259 (1985) 137; err. B276 (1986) 744.

[9] For a recent review on Higgs physics at future hadron and $e^{+} e^{-}$colliders see e.g., A. Djouadi, Int. J. Mod. Phys. A10 (1995) 1.

[10] A. Djouadi, M. Spira and P.M. Zerwas, Phys. Lett. B264 (1991) 440;

S. Dawson, Nucl. Phys. B359 (1991) 283;

M. Spira, D. Graudenz, A. Djouadi and P.M. Zerwas, Nucl. Phys. B453 (1995) 17. 
[11] Proceedings of the Workshop Physics and Experiments with $e^{+} e^{-}$linear colliders, DESY Hamburg, 1996, A. Wagner (ed.) to appear.

[12] J.F. Gunion and H.E. Haber, Phys. Rev. D48 (1993) 5109;

M. Baillargeon, G. Bélanger and F. Boudjema, Phys. Rev. D51 (1995) 4712;

D.L. Borden, V. A. Khoze, W.J. Stirling and J. Ohnemus, Phys. Rev. D50 (1994) 4499;

D.L. Borden, D.A. Bauer and D.O. Caldwell, Phys. Rev. D48 (1993) 4018.

[13] G. Gamberini, G. F. Giudice and G. Ridolfi, Nucl. Phys. B292 (1987) 237;

T. J. Weiler and T. C. Yuan, Nucl. Phys. B318 (1989) 337.

[14] P.H. Chankowski, S. Pokorski, J. Rosiek, Nucl. Phys. B423 (1994) 497.

[15] Particle Data Group (R.M. Barnett et al.), Phys. Rev. D54 (1996) 1.

[16] G. Passarino and M. Veltman, Nucl. Phys. B160 (1979) 151.

[17] G. 't Hooft and M. Veltman, Nucl. Phys. B153 (1979) 365;

A. Denner, Fortschr. Phys. 41 (1993) 4.

[18] A. Djouadi, M. Spira and P.M. Zerwas, Phys. Lett. B276 (1992) 350.

[19] R. Decker, M. Nowakowski and D. Woitschitzky, Phys. Lett. B255 (1991) 605.

[20] H.E. Haber and G. Kane, Phys. Rep. 117 (1985) 75;

J.F. Gunion and H.E. Haber, Nucl. Phys. B272 (1986) 1. 


\section{Figure Captions}

Fig. 1: Feynman diagrams contributing to the Higgs coupling to a photon and a $Z$ boson in the SM (a), additional contributions in the THDM (b) and additional contributions in the $\operatorname{MSSM}(\mathrm{c})$.

Fig. 2: The $W$ and top quark form factors in the SM as a function of the Higgs boson mass (a) and the branching ratios of the decays $H^{0} \rightarrow Z \gamma \rightarrow l^{+} l^{-} \gamma$ and $Z \rightarrow H^{0} \gamma$ in the SM.

Fig. 3: The amplitudes for the contribution of the $W$ boson loop and of the sum of the $t, b$ loops as a function of $M_{h}$ (a) and the contribution of the charged Higgs boson loops as a function of $M_{H^{ \pm}}(\mathrm{b})$. The contributions are in a THDM with $\tan \beta=1.6,5$ and 50 .

Fig. 4: The amplitudes for the contribution of the slepton (a) and squark (except stop) loops (b) as functions of the loop masses for $\tan \beta=1.6,5$ and 50. We have neglected sfermion mixing and have set $M_{A}=1 \mathrm{TeV}$.

Fig. 5: Contours in the $\left(m_{t}^{L R}, m_{\tilde{t}_{1}}\right)$ plane, for which the contribution of the top squark loops to the $h Z \gamma$ coupling is $\left|A_{\tilde{t}}\right|=0.5,0.2$ and 0.1 for $M_{A}=1 \mathrm{TeV}$ (a) and $M_{A}=80$ $\mathrm{GeV}$ (b) with $\tan \beta=1.6$.

Fig. 6: Contours in the $\left(M_{2}, \mu\right)$ plane for $\tan \beta=1.6$ and $M_{A}=1 \mathrm{TeV}$ (a) and $M_{A}=80$ $\mathrm{GeV}$ (b) for which the contribution of the chargino loops to the $h Z \gamma$ coupling is $\left|A_{\chi}\right|=0.2,0.5,1$ and 2 . Also included are the contours for which the lightest chargino mass is $m_{\chi_{1}^{+}}=70$.

Fig. 7: The deviations of the $h \rightarrow Z \gamma$ decay width from the Standard Model value [in \%] for $\tan \beta=1.6$ and the loop masses $m_{i}=100$ and $250 \mathrm{GeV}$. (a) Deviations due to the chargino loops as a function of $M_{2}$ for both signs of $\mu$, and (b) deviations due to the top squark loops as a function of $m_{t}^{L R}$. 

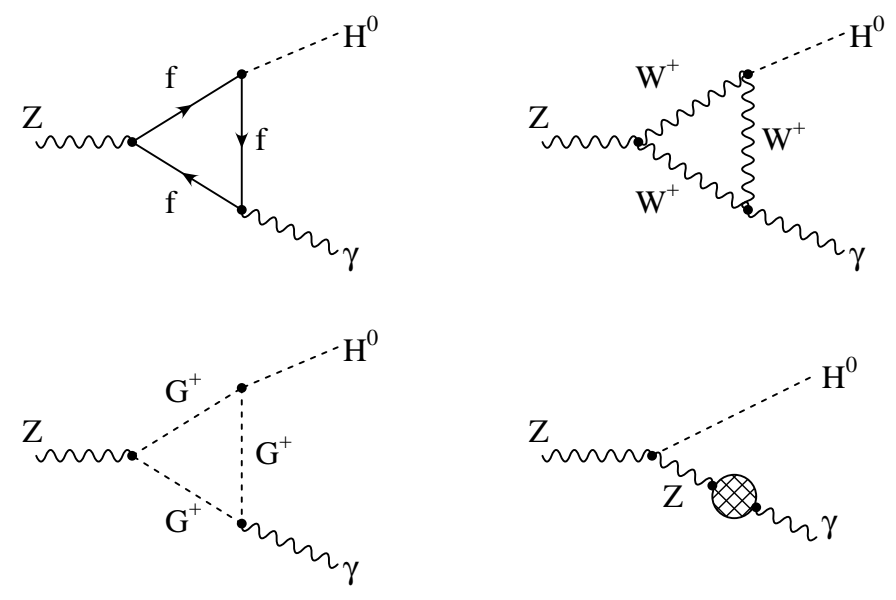

Fig. 1a
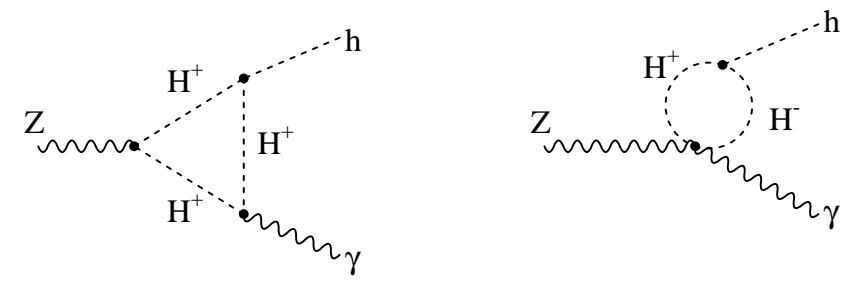

Fig. 1b
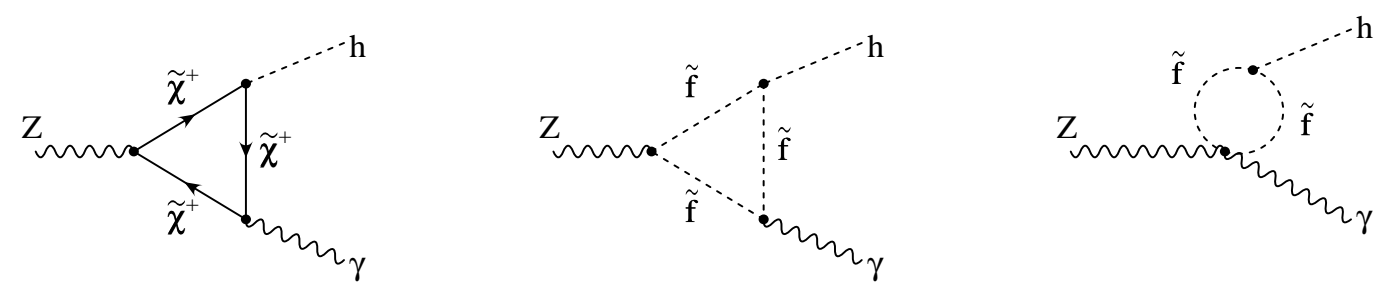

Fig. 1c 

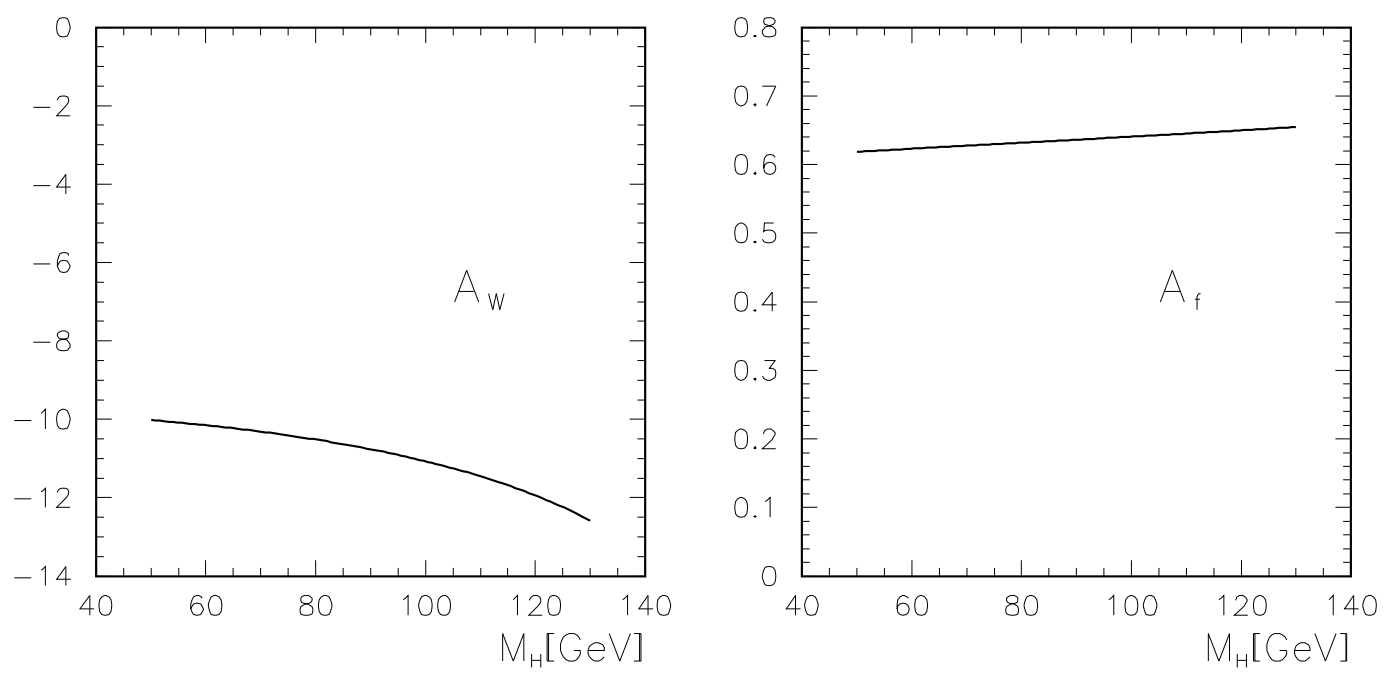

Fig. 2a
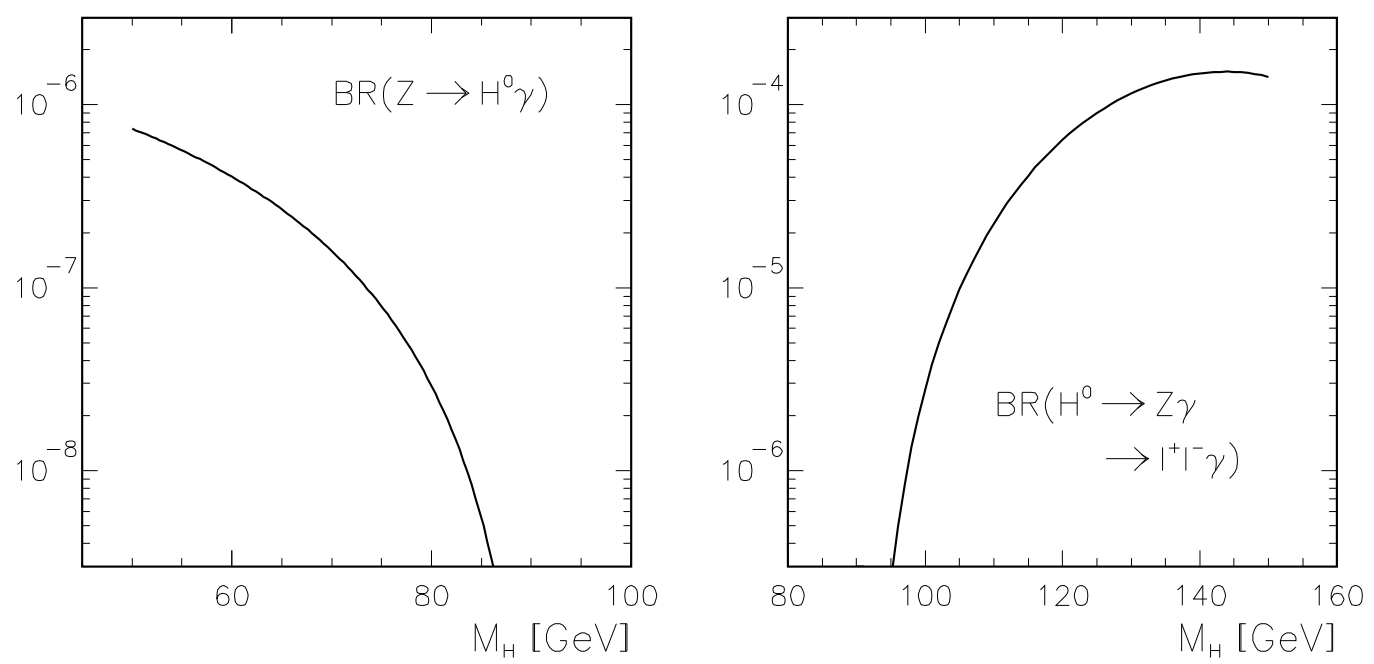

Fig. 2b 

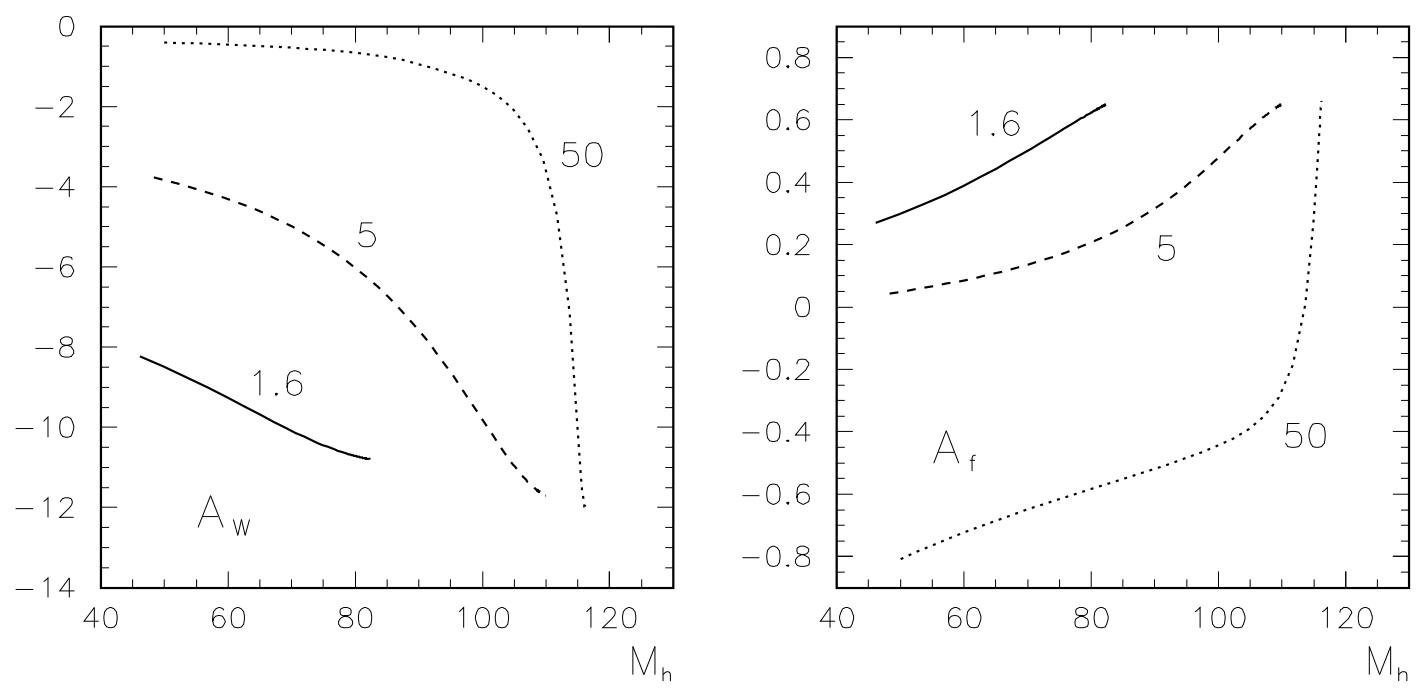

Fig. 3a

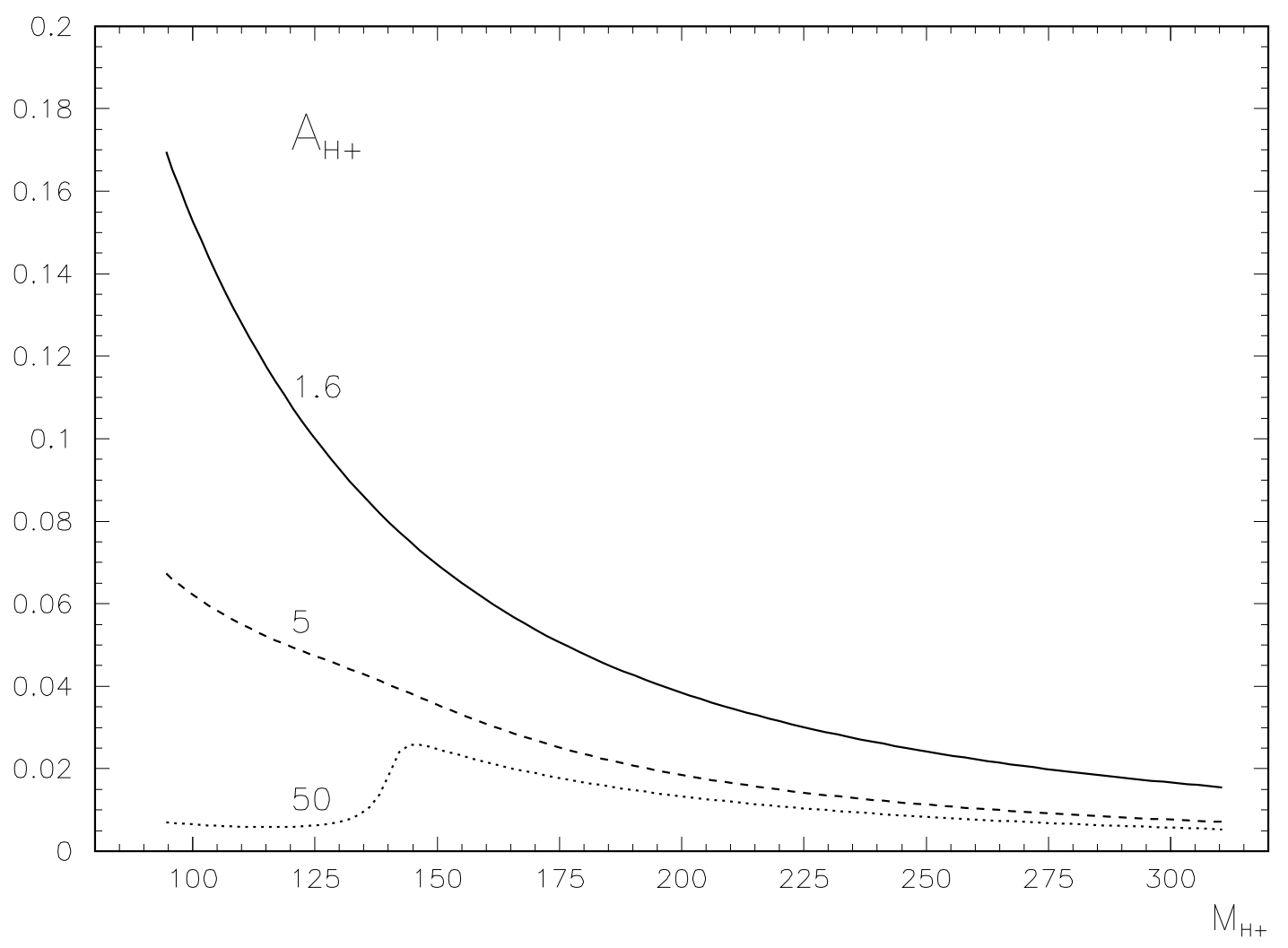

Fig. 3b 


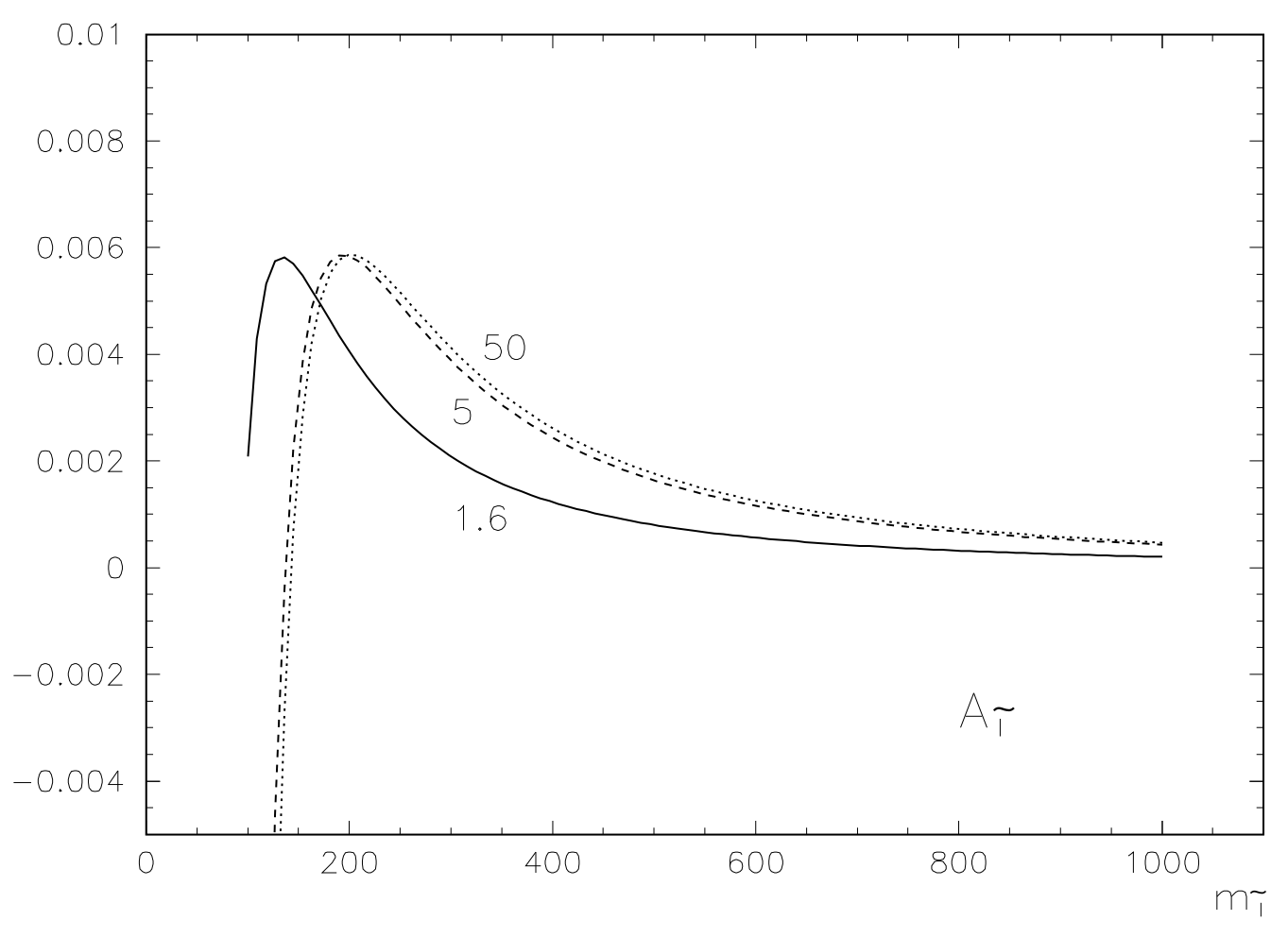

Fig. 4a

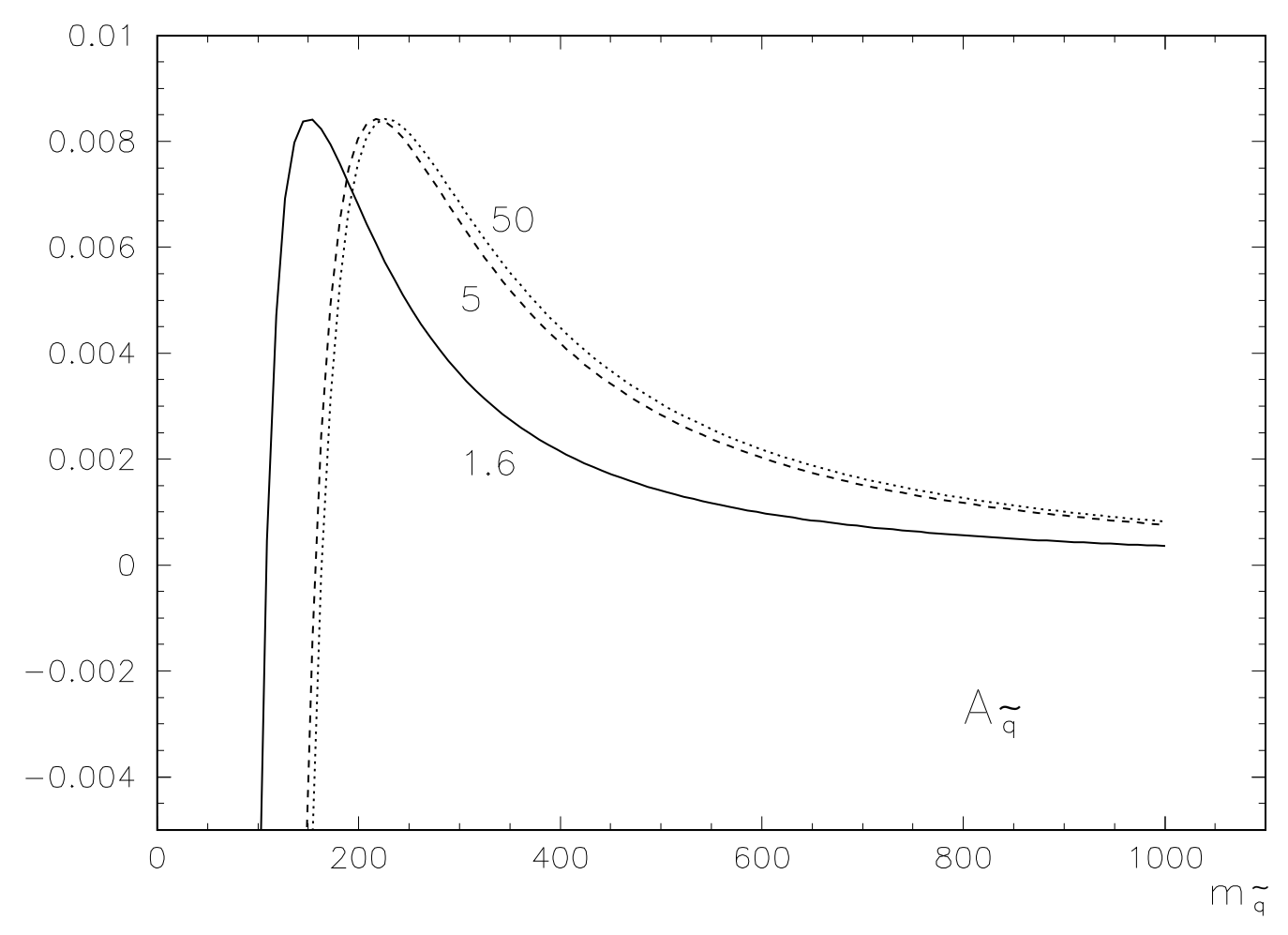

Fig. 4b 


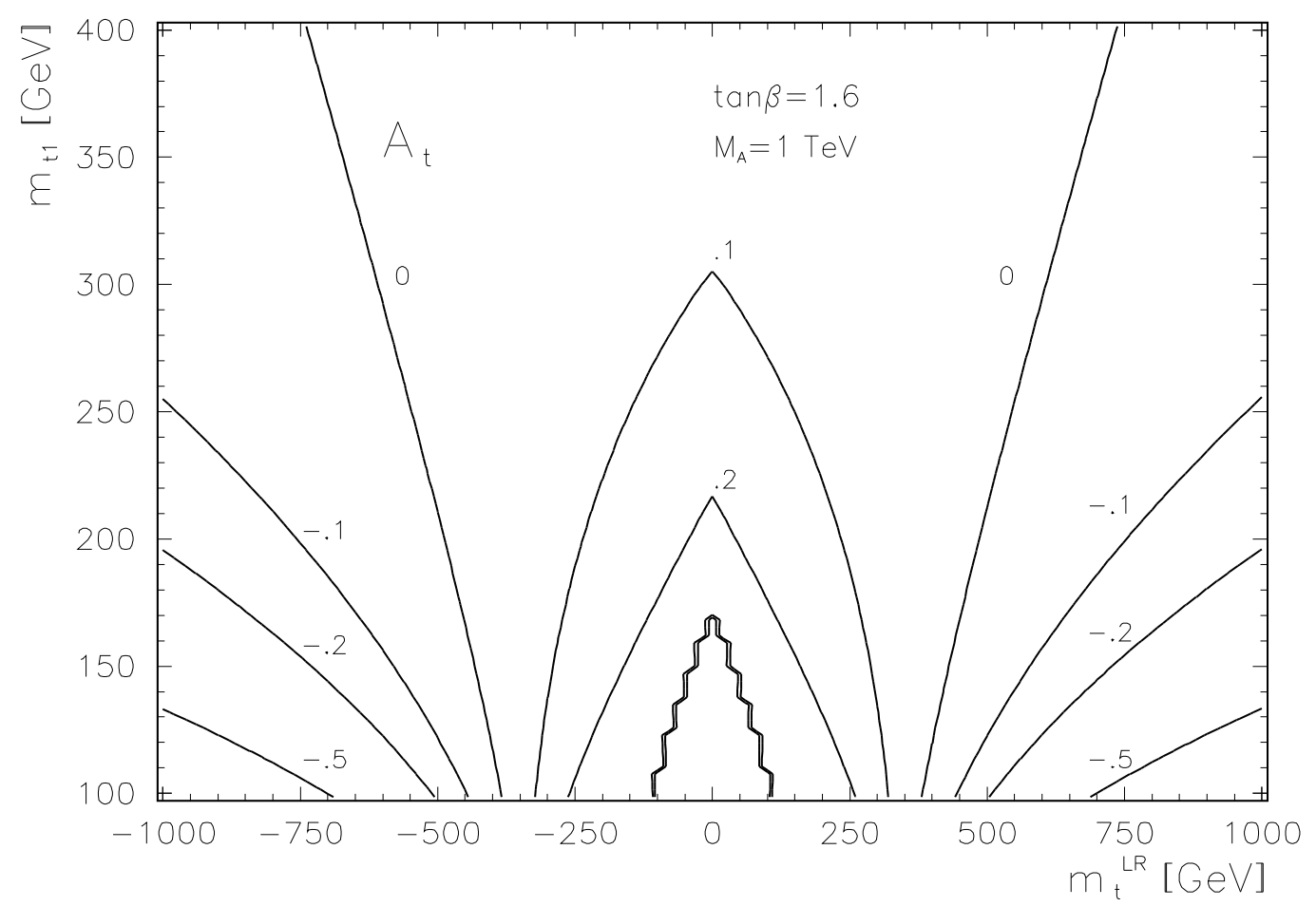

Fig. 5a

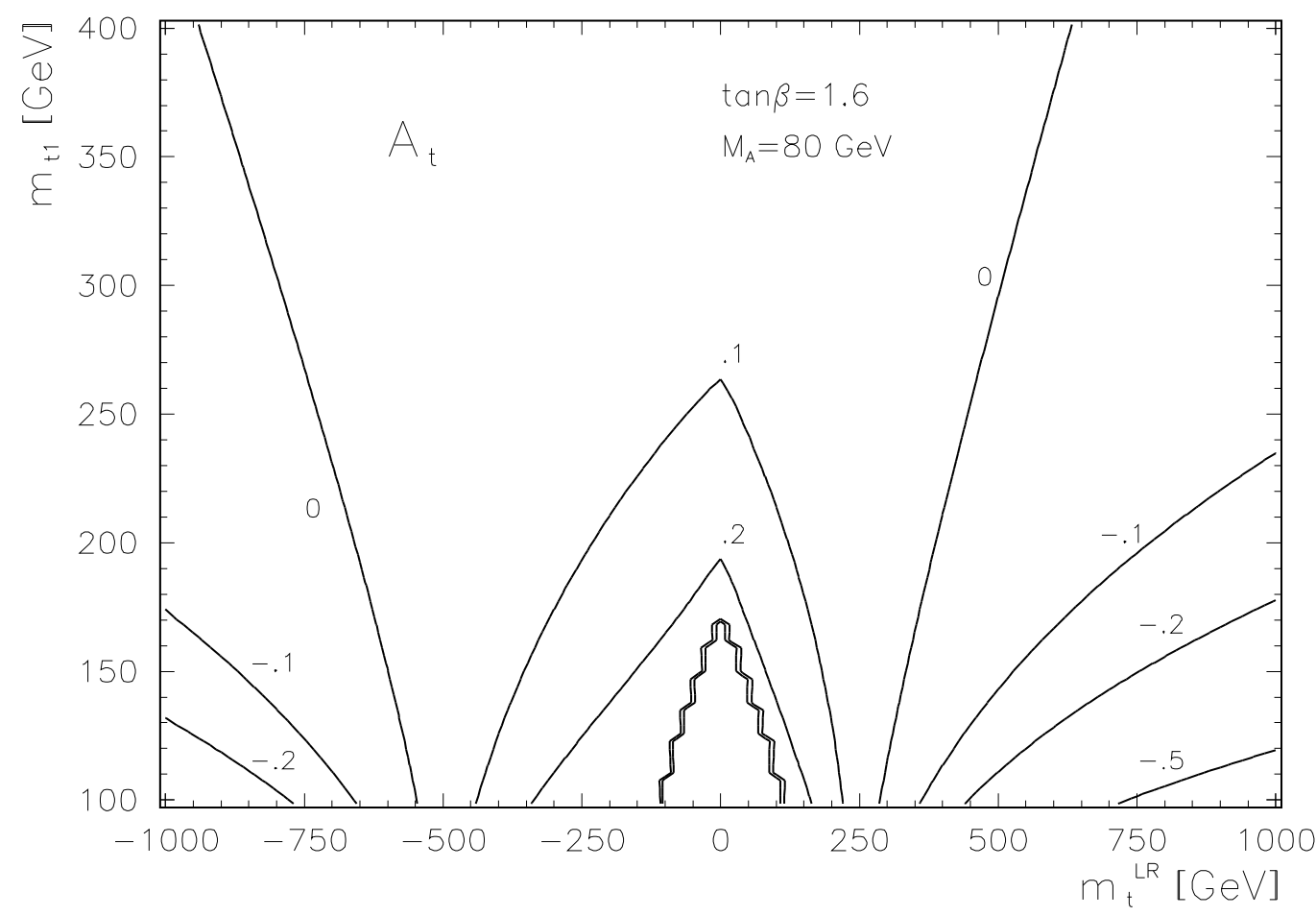

Fig. 5b 


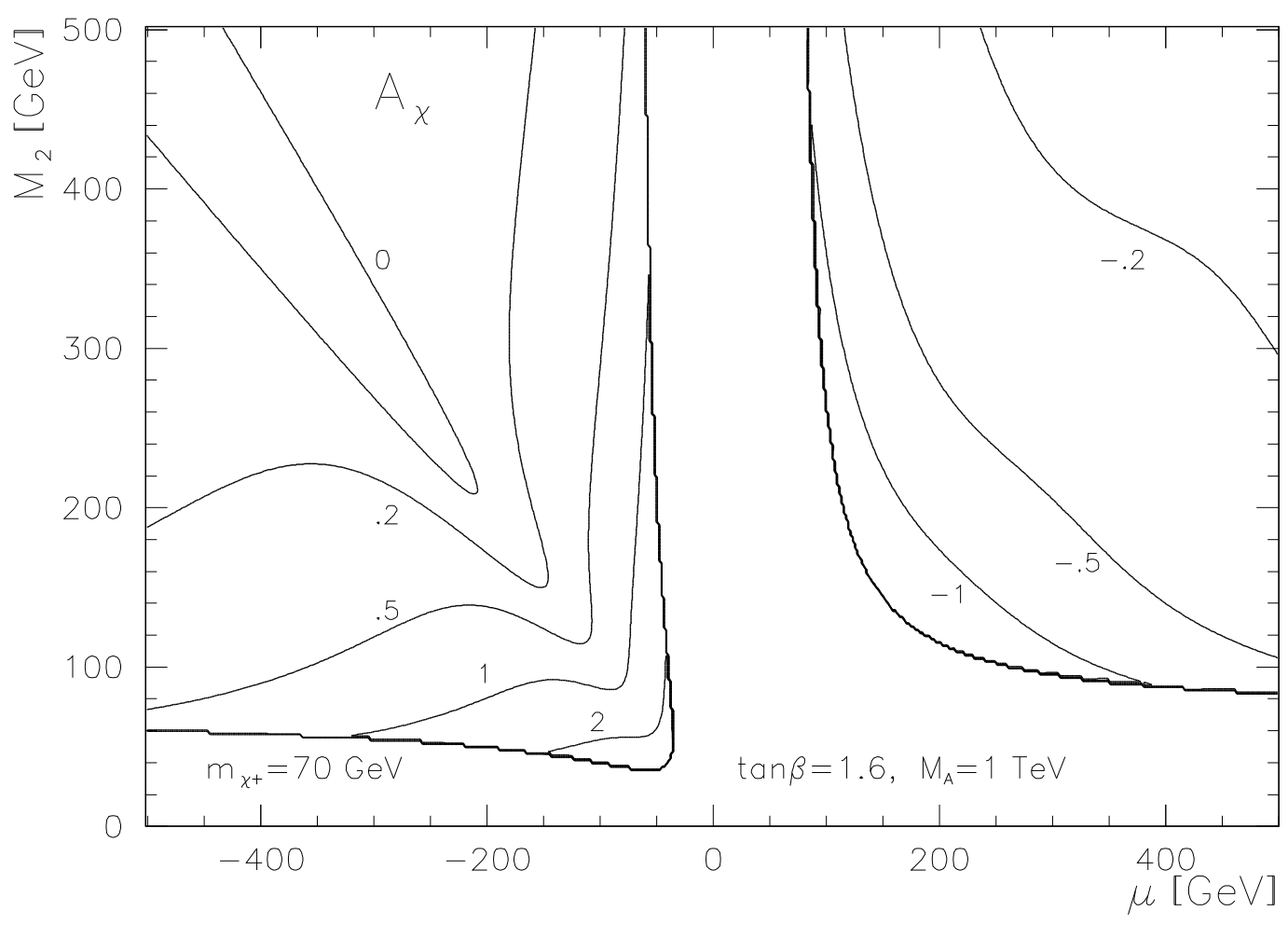

Fig. 6a

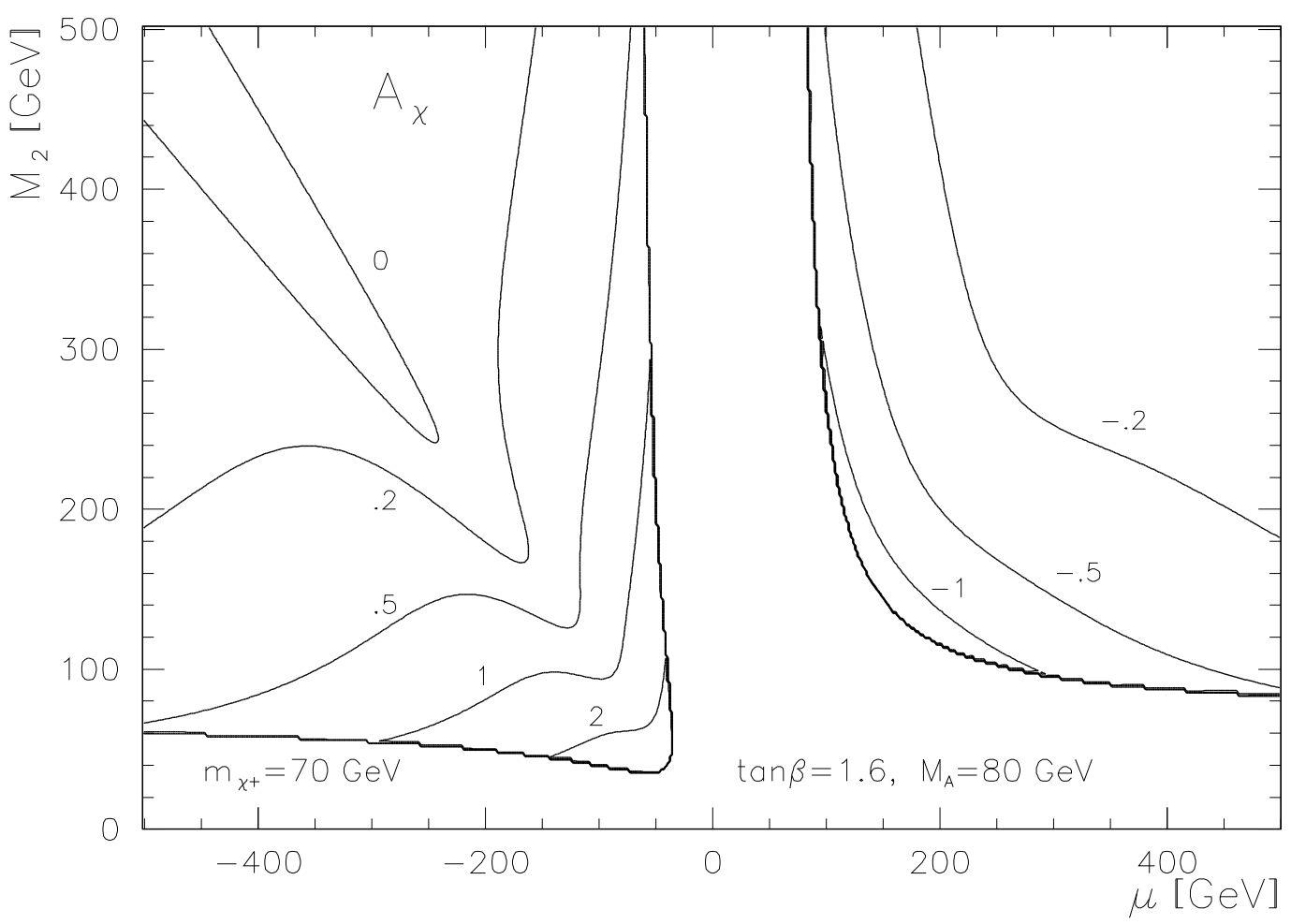

Fig. 6b 

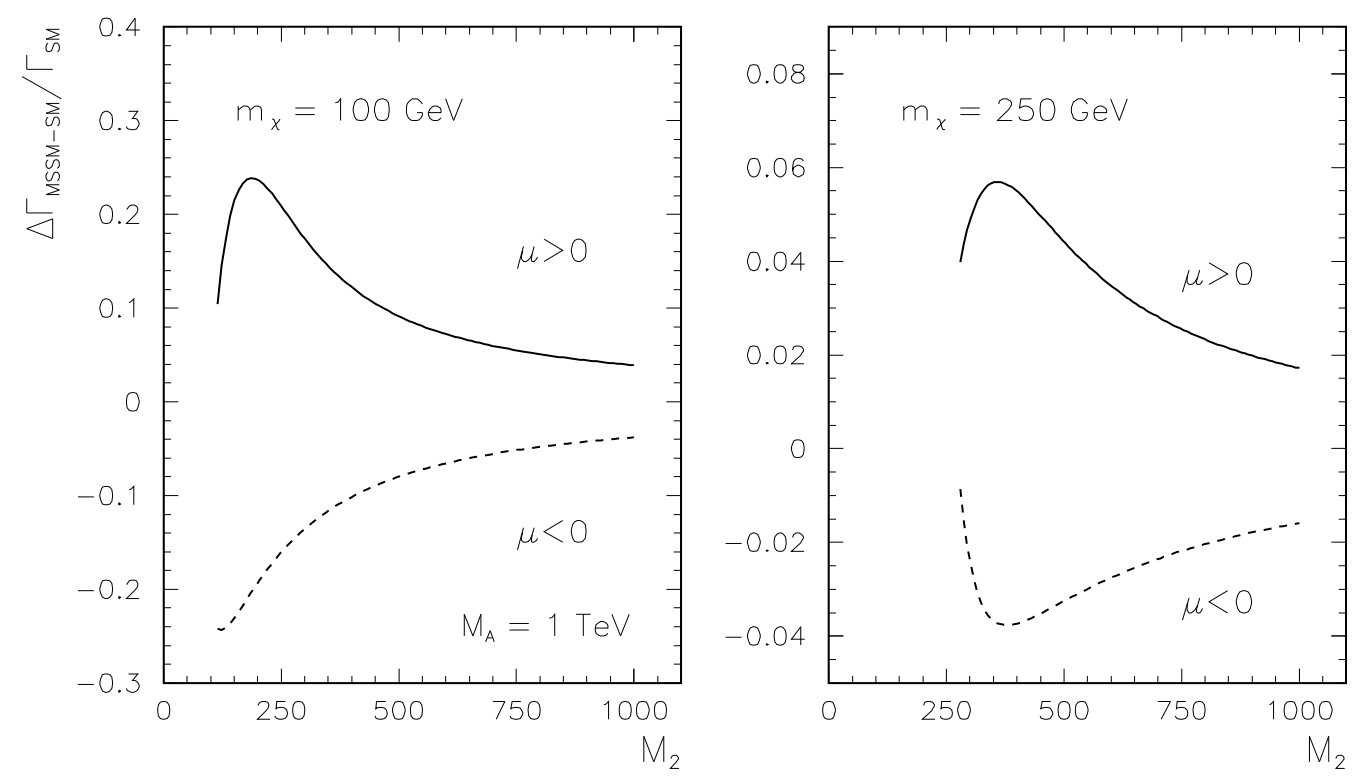

Fig. 7a

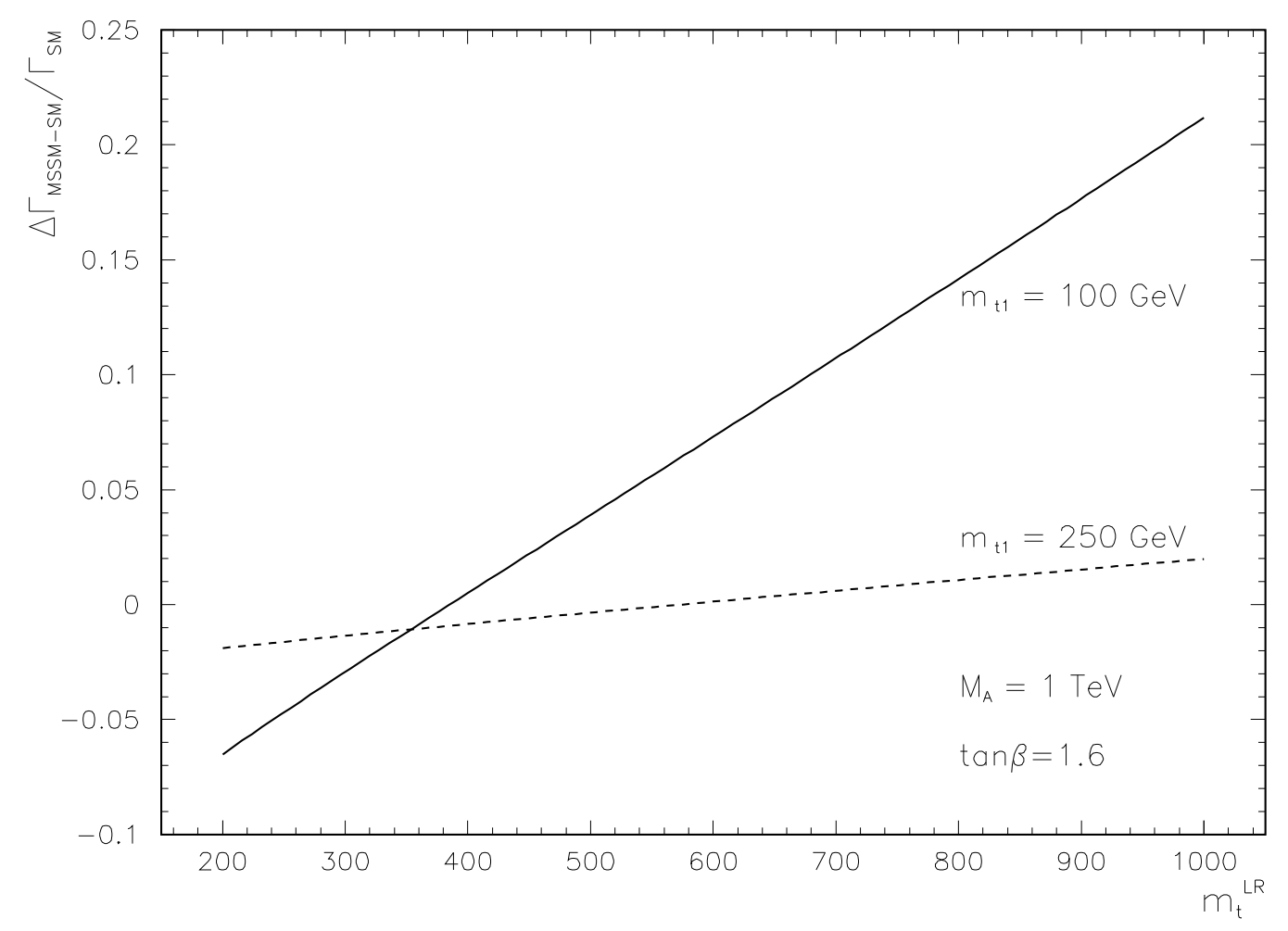

Fig. $7 b$ 